\title{
NUMERICAL METHODS FOR A MULTICOMPONENT TWO-PHASE INTERFACE MODEL WITH GEOMETRIC MEAN INFLUENCE PARAMETERS*
}

\author{
JISHENG $\mathrm{KOU}^{\dagger}$ AND SHUYU SUN $\ddagger$
}

\begin{abstract}
In this paper, we consider an interface model for multicomponent two-phase fluids with geometric mean influence parameters, which is popularly used to model and predict surface tension in practical applications. For this model, there are two major challenges in theoretical analysis and numerical simulation: the first one is that the influence parameter matrix is not positive definite; the second one is the complicated structure of the energy function, which requires us to find out a physically consistent treatment. To overcome these two challenging problems, we reduce the formulation of the energy function by employing a linear transformation and a weighted molar density, and furthermore, we propose a local minimum grand potential energy condition to establish the relation between the weighted molar density and mixture compositions. From this, we prove the existence of the solution under proper conditions and prove the maximum principle of the weighted molar density. For numerical simulation, we propose a modified Newton's method for solving this nonlinear model and analyze its properties; we also analyze a finite element method with a physicalbased adaptive mesh-refinement technique. Numerical examples are tested to verify the theoretical results and the efficiency of the proposed methods.
\end{abstract}

Key words. surface tension, multicomponent fluid, gradient theory, Newton's method, adaptive finite element method

AMS subject classifications. 65N30, 65N50, 49S05

DOI. $10.1137 / 140969579$

1. Introduction. Modeling and simulation of multiphase fluid systems have been a major effort in reservoir engineering [11, 12, 14, 21, 22, 35, 36]. For numericallly simulating the subsurface multiphase flow and transport accurately, it is important to model and compute the surface tension of the multiphase mixture properly since subsurface oil and gas reservoirs typically contain the oil phase, gas phase, and water phase, together with the solid phase (rock or soil) [26]. The capillary effect and its resultant capillary pressure are caused by surface tension between immiscible (or partially miscible) fluids, and it is one of major forces in fluid (oil, gas, and water) flow and transport in the subsurface [22]. In fact, the capillary effect is frequently the leading mechanism of oil recovery in fractured oil reservoirs. In addition to capillary pressure, surface tension also significantly influences other important parameters of porous medium processes, including relative permeability and residual saturations, and thus substantially impacts the flow and the transport of the vapor and liquid phases in a porous medium; consequently, it plays important roles in a number of chemical and reservoir engineering problems [5, 24, 25].

*Submitted to the journal's Computational Methods in Science and Engineering section May 19, 2014; accepted for publication (in revised form) April 20, 2015; published electronically July 16, 2015. This work was supported by the National Natural Science Foundation of China (11301163) and the Key Project of the Chinese Ministry of Education (212109). The authors cheerfully appreciate the generous support of the university research fund to the Computational Transport Phenomena Laboratory at KAUST.

http://www.siam.org/journals/sisc/37-4/96957.html

†School of Mathematics and Statistics, Hubei Engineering University, Xiaogan 432000, Hubei, China (koujisheng@163.com).

${ }_{\ddagger}^{\ddagger}$ Corresponding author. Computational Transport Phenomena Laboratory, Division of Physical Science and Engineering, King Abdullah University of Science and Technology, Thuwal 23955-6900, Kingdom of Saudi Arabia and School of Mathematics and Statistics, Xi'an Jiaotong University, Xi'an 710049, China (shuyu.sun@kaust.edu.sa). 
The gradient theory of two-phase fluid interfaces [10] has been extensively used to model and predict surface tension [5, 10, 24, 25]. Consider a mixture composed of $N(N \geq 2)$ components and denote the mixture composition by $\mathbf{n}=\left[n_{1}, n_{2}, \ldots, n_{N}\right]^{T}$, where $n_{i}$ is the molar density of the $i$ th component. According to the gradient theory, in the absence of an external potential, the Helmholtz energy density of an inhomogeneous fluid is the sum of two contributions: the Helmholtz energy $f_{0}(\mathbf{n})$ of homogeneous fluid at local composition $\mathbf{n}$ and a corrective term (which is a function of the local density gradients):

$$
F(\mathbf{n})=\int_{\Omega}\left(f_{0}(\mathbf{n})+\frac{1}{2} \sum_{i, j=1}^{N} c_{i j} \nabla n_{i} \cdot \nabla n_{j}\right) d \mathbf{x},
$$

where $\Omega$ is a spatial domain, the coefficients $c_{i j}$ are the influence parameters, and $\nabla n_{i}$ represents the local gradient in the $i$ th component density. The Helmholtz free energy density $f_{0}(\mathbf{n})$ of homogeneous fluid is calculated by a thermodynamic model as

$$
\begin{aligned}
f_{0}(\mathbf{n}) & =f_{0}^{\text {ideal }}(\mathbf{n})+f_{0}^{\text {excess }}(\mathbf{n}), \\
f_{0}^{\text {ideal }}(\mathbf{n}) & =R T \sum_{i=1}^{N} n_{i}\left(\ln n_{i}-1\right), \\
f_{0}^{\text {excess }}(\mathbf{n}) & =-n R T \ln (1-b n)+\frac{a(T) n}{2 \sqrt{2} b} \ln \left(\frac{1+(1-\sqrt{2}) b n}{1+(1+\sqrt{2}) b n}\right),
\end{aligned}
$$

where $n=n_{1}+n_{2}+\cdots+n_{N}, T$ is the temperature of the mixture, and $R$ is the universal gas constant. Here, $a(T)$ and $b$ are the energy parameter and the covolume, respectively, and these parameters can be calculated with the mixture composition and the temperature; the detailed computation formulations can be found in the appendix of this paper.

The surface tension $\sigma$ in the two-phase fluid interface is defined by

$$
\sigma(\mathbf{n})=\int_{\Omega}\left(\Phi(\mathbf{n})-\Phi_{B}+\frac{1}{2} \sum_{i, j=1}^{N} c_{i j} \nabla n_{i} \cdot \nabla n_{j}\right) d \mathbf{x},
$$

where $\Phi(\mathbf{n})$ and $\Phi_{B}$ are the grand potentials that will be expressed in section 2 . The surface tension functional (1.5) is closely connected with the Helmholtz free energy (1.1), and their relation will be analyzed in section 2. By the minimum free energy criterion, we can calculate the molar density distribution of each component in the two-phase fluid interface, and then the surface tension is computed by (1.5).

The influence parameter matrix is denoted by $\mathbf{C}=\left(c_{i j}\right)_{i, j=1}^{N}$. The pure component influence parameter $c_{i}$ is calculated by the formulae given in the appendix of this paper. The temperature is constant in the two-phase fluid interface. The dependence of the influence parameters on the component density is neglected [24, 25], and thus their values are independent of spatial positions. The crossed influence parameters $c_{i j}$ is generally described as the modified geometric mean of the pure component influence parameters $c_{i}$ and $c_{j}$ by

$$
c_{i i}=c_{i} \text { and } c_{i j}=\left(1-\beta_{i j}\right) \sqrt{c_{i} c_{j}}, \quad i \neq j,
$$

where the parameters $\beta_{i j}$ are binary interaction coefficients for the influence parameters. The most popular choice strategy of $c_{i j}$ is the geometric mean of $c_{i}$ and $c_{j}$, i.e., 
taking $\beta_{i j}=0$ in (1.6). It has been shown in $[24,25]$ that the choice of $\beta_{i j}=0$ gives the best performance in the surface tension computation of various tested mixtures. So in this paper, we focus on the geometric mean crossed influence parameter model.

The multicomponent two-phase fluid interface model stated above has a similar mathematical formulation to the phase-field models, which have been extensively studied in the recent literature, for example, $[1,3,4,8,16,17,18,27,29,31,32$, $33,37,40]$. However, compared to the conventional phase-field models, there exist at least two key challenges in the multicomponent two-phase fluid interface model considered in this paper. The first challenge is that the geometric mean crossed influence parameter matrix $\mathbf{C}$ is semipositive definite; in fact, it has only one nonzero eigenvalue. Thus, the standard analytical and computational approaches cannot be used directly to deal with this model. The second challenge is that the structure of the energy function is more complicated than the conventional phase-field models. To analyze and simulate this problem, we should find out the measures of the mixture compositions to bound the Helmholtz energy function $f_{0}$ and its derivatives, which is a challenging issue because of the complicated structure of $f_{0}$.

In order to both establish the solution existence of the model and develop efficient numerical simulation methods, the above two key problems must be resolved. To our knowledge, there exist no analytic results for this model. For numerical methods, the path function methods for one-dimensional problems have been developed in the references $[6,9,30]$. The most popular one is the method of selecting the reference component as the path function $[5,10,24,25]$. By the selection of the reference component, the Euler-Lagrange equations can be reduced with the transformations to a solvable system of nonlinear equations, and then the expressions of the surface tension and density distributions are derived. Its principal difficulty is the section of the reference component, which requires the monotonicity of the reference component in the two-phase interface. The selection strategy is usually based on experience, but without any mathematical justification. In our previous work [20], we introduced a weighted molar density instead of reference component, and it is proved to keep the maximum principle in the one-dimensional case, but that proof is based on its monotonicity in one-dimensional space, and thus it cannot be directly extended to multidimensional cases; in this paper, we will develop a new approach to prove its maximum principle in the general multidimensional cases.

The model considered here is also different from the gradient theory model studied in our previous work [19], in which the influence parameter matrix $\mathbf{C}$ is positive definite. In our previous work [19], we developed an efficient computational method to model and calculate the surface tension of single-species two-phase fluid systems based on the rigorous mathematical reformulation of the gradient theory. In the work presented in this paper, we will resolve the abovementioned two key problems in the multicompoent two-phase interface model with geometric mean influence parameters, and from this, we will establish the solution existence of the model and develop efficient numerical simulation methods. The main ingredients of our contributions are listed below:

(1) By using a linear transformation and a weighted molar density, we simplify the formulation of the energy function $F$ for the convenience of analysis and prove that the weighted molar density satisfies the maximum principle.

(2) The local minimum grand potential energy condition is proposed to establish the relation between the weighted density and mixture compositions, which allows us to calculate the mixture compositions once the weighted density is given. 
(3) The boundedness of $f_{0}$ and its derivatives is obtained by coupling the maximum principle of the weighted density and the local minimum grand potential energy condition.

(4) Based on the above approaches, the existence of the minimizer of $F$ is established.

(5) An efficient modified Newton's method is developed to solve this nonlinear model, and it is also analyzed in theory.

(6) We analyze a finite element method with a physical-based adaptive meshrefinement technique.

In addition, we test some numerical examples to verify theoretical results and the efficiency of the proposed methods.

The rest of this paper is organized as follows. In section 2, we will introduce the weighted molar density and the local grand potential energy condition and then carry out the mathematical analysis of the model. In section 3, we will develop and analyze the modified Newton's method and the adaptive finite element method for simulating the model problem. In section 4, we will give some numerical results for hydrocarbon mixtures. Finally, concluding remarks are provided in section 5 .

2. Mathematical analysis for the multicomponent two-phase interface model. In this section, we will first describe the original formulation of the multicomponent two-phase interface model, and then using a linear transformation, we will simplify the model to a convenient formulation. Furthermore, by using the weighted molar density, we introduce the local minimum grand potential energy condition to establish the relation between the weighted density and mixture compositions, and from this, we can carry out the mathematical analysis for this model, including the existence of the minimizer and maximum principle.

2.1. Original formulation of the interface model. Suppose that $\Omega$ is an open, bounded, and connected domain containing the two-phase fluid interface, and the boundary $\partial \Omega$ is sufficiently smooth. Moreover, both gas and liquid regions within $\Omega$ are not empty. Let $V=\left(H^{1}(\Omega)\right)^{N}$ be the functional space associated with the norm

$$
\|\mathbf{v}\|_{V}^{2}=\|\mathbf{v}\|_{\left(L^{2}(\Omega)\right)^{N}}^{2}+\sum_{i=1}^{N}\left\|\nabla v_{i}\right\|_{\left(L^{2}(\Omega)\right)^{d}}^{2}, \quad \mathbf{v} \in V,
$$

where $\mathbf{v}=\left[v_{1}, \ldots, v_{N}\right]^{T}$ and $N$ is the number of total components of a mixture.

According to the second law of thermodynamics [13], the Helmholtz free energy of a system at equilibrium should attain its minimum. Consider the constrained minimization problem: find $\mathbf{n} \in V$ satisfying

$$
F(\mathbf{n})=\min _{\widetilde{\mathbf{n}} \in V} F(\widetilde{\mathbf{n}})
$$

subject to

$$
\int_{\Omega} \mathbf{n} d \mathbf{x}=\mathbf{n}^{t}
$$

Here, $\mathbf{n}^{t}=\left[n_{1}^{t}, n_{2}^{t}, \ldots, n_{N}^{t}\right]^{T}$ is a given constant vector representing the fixed amount of given substance. The constraint condition (2.2) ensures the mass balance of each component in the domain. The appropriate admissible class is defined as

$$
V^{C}=\left\{\mathbf{n} \in\left(H^{1}(\Omega)\right)^{N}: \quad \int_{\Omega} \mathbf{n} d \mathbf{x}=\mathbf{n}^{t}\right\} .
$$

Apparently, $\mathbf{n}=\frac{\mathbf{n}^{t}}{|\Omega|} \in V^{C}$, so $V^{C}$ is nonempty. 
2.2. Transformation of the original variables. The standard analytical and computational approaches cannot be used directly to deal with the original formulation because the geometric mean crossed influence parameter matrix $\mathbf{C}$ is not positive definite. The following linear transformation [20] will be used to transform the original formulation to a convenient form. The influence matrix is rewritten as $\mathbf{C}=\mathbf{c c}^{T}$, where $\mathbf{c}=\left[\sqrt{c_{1}}, \ldots, \sqrt{c_{N}}\right]^{T}$. Denote the unique nonzero eigenvalue of $\mathbf{C}$ by $\lambda=\mathbf{c}^{T} \mathbf{c}=$ $\sum_{i=1}^{N} c_{i}$, and its corresponding eigenvector $\mathbf{v}_{1}=\frac{\mathbf{c}}{\sqrt{\lambda}}=\frac{1}{\sqrt{\sum_{i=1}^{N} c_{i}}}\left[\sqrt{c_{1}}, \ldots, \sqrt{c_{N}}\right]^{T}$.

Since $\mathbf{C}$ is a symmetrical matrix, there exist $N-1$ zero eigenvalues of $\mathbf{C}$, and the corresponding eigenvectors, denoted by $\mathbf{v}_{i}, \quad 2 \leq i \leq N$. We choose $\mathbf{v}_{i}$ as

$$
\begin{aligned}
& \mathbf{v}_{i}(1)=\frac{\sqrt{c_{i}}}{\sqrt{\lambda}}, \quad \mathbf{v}_{i}(i)=-\frac{\sqrt{c_{1}}}{\sqrt{\lambda}}, \quad 2 \leq i \leq N, \\
& \mathbf{v}_{i}(j)=0, \quad 2 \leq i \leq N, \quad 2 \leq j \leq N, \quad j \neq i .
\end{aligned}
$$

It is followed by the above definition that $\mathbf{C v}_{i}=\mathbf{c c}^{T} \mathbf{v}_{i}=0$ and $\mathbf{v}_{i}^{T} \mathbf{v}_{1}=0$ for $2 \leq i \leq N$. Thus, the vector group $\left\{\mathbf{v}_{i}\right\}_{i=1}^{N}$ is linearly independent.

We now define $\mathbf{Q}=\left[\mathbf{v}_{1}, \ldots, \mathbf{v}_{N}\right]$ as a transformation matrix, which is invertible. Furthermore, we define a vector $\mathbf{u}=\left[u_{1}, u_{2}, \ldots, u_{N}\right]^{T}$ as

$$
\mathbf{u}=\mathbf{Q}^{-1} \mathbf{n}, \quad \mathbf{n}=\mathbf{Q} \mathbf{u} .
$$

For sake of notation simplification, we drop the subscript of $u_{1}$; that is, let $u=u_{1}$. The expression of $u$ is calculated as

$$
u=\mathbf{v}_{1}^{T} \mathbf{n}=\frac{1}{\sqrt{\lambda}} \sum_{i=1}^{N} \sqrt{c_{i}} n_{i} .
$$

The variable $u$ is called the weighted molar density of a mixture. In our previous work [20], we have proved the maximum principle of $u$ by its monotonicity in the one-dimensional case, but that proof cannot be directly extended to multidimensional cases; in this paper, it will be proved in Theorem 2.3 that this maximum principle holds for the general multidimensional cases. This property plays an important role in the boundedness of $f_{0}$ and its derivatives. From the above definitions, we have the following relations:

$$
\begin{aligned}
& \frac{\partial n_{1}}{\partial u}=\frac{\sqrt{c_{1}}}{\sqrt{\lambda}}, \quad \frac{\partial n_{1}}{\partial u_{i}}=\frac{\sqrt{c_{i}}}{\sqrt{\lambda}}, \quad 2 \leq i \leq N, \\
& \frac{\partial n_{i}}{\partial u}=\frac{\sqrt{c_{i}}}{\sqrt{\lambda}}, \quad \frac{\partial n_{i}}{\partial u_{i}}=-\frac{\sqrt{c_{1}}}{\sqrt{\lambda}}, \quad 2 \leq i \leq N, \\
& \frac{\partial n_{i}}{\partial u_{j}}=0, \quad 2 \leq i, j \leq N, \quad i \neq j .
\end{aligned}
$$

It is noted that $\mathbf{n}$ and $\mathbf{u}$ are the variables in space, but the transformation matrix $\mathbf{Q}$ is independent of spatial positions. For the regularity relation between $\mathbf{u}$ and $\mathbf{n}$, we have the following lemma.

LEMmA 2.1. If $\mathbf{u} \in\left(H^{s}(\Omega)\right)^{N}(s \geq 0)$, then $\mathbf{n} \in\left(H^{s}(\Omega)\right)^{N}$, and vice versa.

Proof. The proof can be obtained immediately from the property of the linear transformation $\mathbf{Q}$. 
2.3. Simplified formulation of the interface model and theoretical analysis. We now use the above linear transformation to simplify the original model formulation to a convenient form. Furthermore, we introduce the local minimum grand potential energy condition. From this, we prove the existence of the minimizer and analyze the connection between the surface tension functional and the Helmholtz free energy.

Denote $g_{0}(\mathbf{u})=f_{0}(\mathbf{Q u})$. Using the relations given by (2.5)-(2.7), we get $\sum_{i, j=1}^{N} c_{i j}$ $\nabla n_{i} \cdot \nabla n_{j}=\lambda|\nabla u|^{2}$. Denote $G(\mathbf{u})=F(\mathbf{n})=F(\mathbf{Q u})$, and thus the Helmholtz energy density (1.1) of an inhomogeneous fluid is simplified as

$$
G(\mathbf{u})=\int_{\Omega}\left(g_{0}(\mathbf{u})+\frac{1}{2} \lambda|\nabla u|^{2}\right) d \mathbf{x} .
$$

Let $\mathbf{u}^{t}=\mathbf{Q}^{-1} \mathbf{n}^{t}$, and then the constraint (2.2) becomes

$$
\int_{\Omega} \mathbf{u} d \mathbf{x}=\mathbf{u}^{t}
$$

In order to introduce the local minimum grand potential energy condition, we first need to define the chemical potential and grand potential, which are also related to the surface tension. The chemical potential $\mu_{i}^{0}$ of component $i$ is defined as

$$
\mu_{i}^{0}=\left(\frac{\partial f_{0}(\mathbf{n})}{\partial n_{i}}\right)_{T, n_{1}, \ldots, n_{i-1}, n_{i+1}, \ldots, n_{N}} .
$$

Denote by $\mu_{i}$ the value of the chemical potential $\mu_{i}^{0}$ in the equilibrium bulk phases. In the two-phase interface region, $\mu_{i}^{0}$ is generally not equal to $\mu_{i}$. We note that $\mu_{i}$ can also be viewed as a set of Lagrange multipliers due to the constraint (2.2) when we use the method of Lagrange multipliers to calculate the minimizer of (2.1) under this constraint, and it is proved in [19] by the principle of variational calculus that $\mu_{i}$ is the chemical potential of the $i$ th component in the equilibrium bulk phases.

The grand potential is defined as

$$
\Phi(\mathbf{n})=f_{0}(\mathbf{n})-\sum_{i=1}^{N} \mu_{i} n_{i} .
$$

Denote by $\Phi_{B}$ the value of $\Phi(\mathbf{n})$ in the equilibrium bulk phases, and in the two-phase interface region, $\Phi(\mathbf{n})$ is generally greater than $\Phi_{B}$. Let $\mathbf{n}^{G}$ and $\mathbf{n}^{L}$ be the equilibrium bulk compositions of gas and liquid phases, respectively. For $\Phi_{B}$ and $\mu_{i}$, let us state the following physical properties of the grand potential and the chemical potential, which are assumed to hold in this paper:

(S1) The grand potential $\Phi$ attains its minimum $\Phi_{B}$ at the equilibrium bulk gas and liquid phase regions, i.e., $\Phi_{B}=\Phi\left(\mathbf{n}^{G}\right)=\Phi\left(\mathbf{n}^{L}\right)$.

(S2) The chemical potential of each component satisfies $\mu_{i}=\mu_{i}^{0}\left(\mathbf{n}^{G}\right)=\mu_{i}^{0}\left(\mathbf{n}^{L}\right)$ at the equilibrium bulk phases.

Remark 1. We now give some remarks on the validity of the above physical assumptions (S1) and (S2). For the sharp interface problem, which is like the zerodimensional case of the above framework, we consider the following energy minimum of

$$
H=f_{0}\left(\mathbf{n}^{G}\right) V^{G}+f_{0}\left(\mathbf{n}^{L}\right) V^{L}
$$

Copyright (C) by SIAM. Unauthorized reproduction of this article is prohibited. 
subject to

$$
\mathbf{n}^{G} V^{G}+\mathbf{n}^{L} V^{L}=\mathbf{N}^{t}, \quad V^{G}+V^{L}=V^{t} .
$$

Substituting the constraints into (2.11), we get

$$
H\left(\mathbf{N}^{G}, V^{G}\right)=f_{0}\left(\frac{\mathbf{N}^{G}}{V^{G}}\right) V^{G}+f_{0}\left(\frac{\mathbf{N}^{t}-\mathbf{N}^{G}}{V^{t}-V^{G}}\right)\left(V^{t}-V^{G}\right),
$$

where $\mathbf{N}^{G}=\mathbf{n}^{G} V^{G}$ and $\mathbf{N}^{L}=\mathbf{n}^{L} V^{L}$. The partial derivatives of $H$ are calculated as

$$
\begin{aligned}
\frac{\partial H\left(\mathbf{N}^{G}, V^{G}\right)}{\partial N_{i}^{G}}= & \mu_{i}^{0}\left(\mathbf{n}^{G}\right) V^{G} \frac{\partial\left(\frac{N_{i}^{G}}{V^{G}}\right)}{\partial N_{i}^{G}}+\mu_{i}^{0}\left(\mathbf{n}^{L}\right)\left(V^{t}-V^{G}\right) \frac{\partial\left(\frac{N_{i}^{t}-N_{i}^{G}}{V^{t}-V^{G}}\right)}{\partial N_{i}^{G}} \\
= & \mu_{i}^{0}\left(\mathbf{n}^{G}\right)-\mu_{i}^{0}\left(\mathbf{n}^{L}\right), \\
\frac{\partial H\left(\mathbf{N}^{G}, V^{G}\right)}{\partial V^{G}}= & f_{0}\left(\mathbf{n}^{G}\right)+\sum_{i=1}^{N} \mu_{i}^{0}\left(\mathbf{n}^{G}\right) V^{G} \frac{\partial\left(\frac{N_{i}^{G}}{V^{G}}\right)}{\partial V^{G}} \\
& -f_{0}\left(\mathbf{n}^{L}\right)+\sum_{i=1}^{N} \mu_{i}^{0}\left(\mathbf{n}^{L}\right)\left(V^{t}-V^{G}\right) \frac{\partial\left(\frac{N_{i}^{t}-N_{i}^{G}}{V^{t}-V^{G}}\right)}{\partial V^{G}} \\
= & f_{0}\left(\mathbf{n}^{G}\right)-\sum_{i=1}^{N} \mu_{i}^{0}\left(\mathbf{n}^{G}\right) n_{i}^{G}-f_{0}\left(\mathbf{n}^{L}\right)+\sum_{i=1}^{N} \mu_{i}^{0}\left(\mathbf{n}^{L}\right) n_{i}^{L} .
\end{aligned}
$$

Following the minimum conditions $\frac{\partial H\left(\mathbf{N}^{G}, V^{G}\right)}{\partial N_{i}^{G}}=\frac{\partial H\left(\mathbf{N}^{G}, V^{G}\right)}{\partial V^{G}}=0$, we derive from (2.14) and (2.15) that $\mu_{i}^{0}\left(\mathbf{n}^{G}\right)=\mu_{i}^{0}\left(\mathbf{n}^{L}\right)$ and $\Phi\left(\mathbf{n}^{G}\right)=\Phi\left(\mathbf{n}^{L}\right)$ at the equilibrium bulk phases. These two equilibrium conditions also apply to one-dimensional scenarios, because of the flat interface between two phases. In fact, we first multiply by $\frac{d n_{i}}{d x}$ both sides of the Euler-Lagrange equations (2.38) described in section 2.4 and then sum it by $i$ from 1 to $N$ to reach

$$
\sum_{i, j=1}^{N} c_{i j} \frac{d^{2} n_{j}}{d x^{2}} \frac{d n_{i}}{d x}=\sum_{i=1}^{N}\left(\mu_{i}^{0}-\mu_{i}\right) \frac{d n_{i}}{d x} .
$$

Let $\Omega$ be an interval containing two bulk equilibrium phases and a two-phase interface. Moreover, assume that the left-hand side is the bulk gas end and the right-hand side is the bulk liquid end. We integrate (2.16) over a subinterval $\left(x_{1}, x_{2}\right) \subseteq \Omega$ as

$$
\int_{x_{1}}^{x_{2}} \sum_{i, j=1}^{N} c_{i j} \frac{d^{2} n_{j}}{d x^{2}} \frac{d n_{i}}{d x} d x=\int_{x_{1}}^{x_{2}} \sum_{i=1}^{N}\left(\mu_{i}^{0}-\mu_{i}\right) \frac{d n_{i}}{d x} d x .
$$

Since

$$
\int_{x_{1}}^{x_{2}} \sum_{i, j=1}^{N} c_{i j} \frac{d^{2} n_{j}}{d x^{2}} \frac{d n_{i}}{d x} d x=\left.\sum_{i, j=1}^{N} c_{i j} \frac{d n_{j}}{d x} \frac{d n_{i}}{d x}\right|_{x_{1}} ^{x_{2}}-\int_{x_{1}}^{x_{2}} \sum_{i, j=1}^{N} c_{i j} \frac{d^{2} n_{i}}{d x^{2}} \frac{d n_{j}}{d x} d x
$$

for the left-hand side of (2.17), we get

$$
\int_{x_{1}}^{x_{2}} \sum_{i, j=1}^{N} c_{i j} \frac{d^{2} n_{j}}{d x^{2}} \frac{d n_{i}}{d x} d x=\left.\frac{1}{2} \sum_{i, j=1}^{N} c_{i j} \frac{d n_{j}}{d x} \frac{d n_{i}}{d x}\right|_{x_{1}} ^{x_{2}} .
$$

Copyright $@$ by SIAM. Unauthorized reproduction of this article is prohibited. 
On the other hand, the right-hand side of (2.17) is calculated as

$$
\begin{aligned}
\int_{x_{1}}^{x_{2}} \sum_{i=1}^{N}\left(\mu_{i}^{0}-\mu_{i}\right) \frac{d n_{i}}{d x} d x & =\int_{x_{1}}^{x_{2}} \sum_{i=1}^{N}\left(\mu_{i}^{0}-\mu_{i}\right) d n_{i} \\
& =\int_{x_{1}}^{x_{2}} d \Phi=\Phi\left(\mathbf{n}\left(x_{2}\right)\right)-\Phi\left(\mathbf{n}\left(x_{1}\right)\right) .
\end{aligned}
$$

It is deduced from (2.17), (2.19), and (2.20) that

$$
\left.\frac{1}{2} \sum_{i, j=1}^{N} c_{i j} \frac{d n_{j}}{d x} \frac{d n_{i}}{d x}\right|_{x_{1}} ^{x_{2}}=\Phi\left(\mathbf{n}\left(x_{2}\right)\right)-\Phi\left(\mathbf{n}\left(x_{1}\right)\right) .
$$

Taking $x_{1}$ and $x_{2}$ within the bulk equilibrium gas and liquid phase regions, respectively, and taking into account $\frac{d n_{i}}{d x}=0$ in the bulk phases, we obtain $\Phi\left(\mathbf{n}^{G}\right)=\Phi\left(\mathbf{n}^{L}\right)$ from (2.21). For two-dimensional and three-dimensional cases, the interface between the two phases might not be flat, which could lead to $\Phi\left(\mathbf{n}^{G}\right) \neq \Phi\left(\mathbf{n}^{L}\right)$, even through $\mu_{i}^{0}\left(\mathbf{n}^{G}\right)=\mu_{i}^{0}\left(\mathbf{n}^{L}\right)$ still holds. However, if the interface tension effect is small, these two assumptions above (S1) and (S2) may still hold approximately. Finally, we note that for the one-dimensional case, it has been proved in [20] that

$$
\lambda\left(\frac{d u}{d x}\right)^{2}=2\left(\Phi(\mathbf{n})-\Phi_{B}\right) .
$$

This shows that $\Phi(\mathbf{n}) \geq \Phi_{B}$ at the two-phase fluid interface.

Denote $\mathbf{u}^{G}=\mathbf{Q}^{-1} \mathbf{n}^{G}$ and $\mathbf{u}^{L}=\mathbf{Q}^{-1} \mathbf{n}^{L}$. It will be proved in Theorem 2.3 that the weighted density $u$ satisfies $u^{G} \leq u \leq u^{L}$ within the two-phase fluid interface. Let $\boldsymbol{\mu}=\left[\mu_{1}, \ldots, \mu_{N}\right]^{T}$. For any given scalar value $u \in\left[u^{G}, u^{L}\right]$, we define an energy function of $\left(u_{2}, \ldots, u_{N}\right)$ as

$$
q\left(u_{2}, \ldots, u_{N} ; u\right)=q\left(u_{2}, \ldots, u_{N}\right)=\Phi(\mathbf{Q u})-\Phi_{B}=g_{0}(\mathbf{u})-\boldsymbol{\mu}^{T} \mathbf{Q u}-\Phi_{B} .
$$

From the property of the grand potential, we know that $q\left(u_{2}, \ldots, u_{N} ; u\right) \geq 0$ for $u \in\left[u^{G}, u^{L}\right]$. In order to analyze the transformed minimizing problem (2.8) with the constraint (2.9), we propose the following local minimum grand potential energy condition.

Condition $(A)$. For any given $u \in\left[u^{G}, u^{L}\right]$, there exists a unique $\left(u_{2}, \ldots, u_{N}\right)$ such that

$$
q\left(u_{2}, \ldots, u_{N} ; u\right)=\min _{\widetilde{u}_{i}, i=2, \ldots, N} q\left(\widetilde{u}_{2}, \ldots, \widetilde{u}_{N} ; u\right) .
$$

This condition will be verified in numerical tests. In what follows, Condition (A) is always assumed to be true for the model considered in this paper, and this condition will also be used throughout the numerical tests to be carried out in section 4 . Condition (A) implies that $u_{i}, i=2, \ldots, N$, can be viewed as the functions of $u$, and thus denote $u_{i}=\psi_{i}(u)$. We assume that the function $\psi_{i}$ is differentiable with respect to $u$ and its derivative is bounded in $\left[u^{G}, u^{L}\right]$. From this, the function $f_{0}$ and its partial derivatives $\mu_{i}^{0}$ are uniformly bounded.

From Condition (A), we claim that for any given function $u \in L^{2}(\Omega)$ satisfying $u^{G} \leq u \leq u^{L}$ and the constraint $(2.9)$, there exists a unique $\left(u_{2}, \ldots, u_{N}\right)$ defined on $\Omega$ such that

$$
\min _{u_{i} \in L^{2}(\Omega), i=2, \ldots, N} \int_{\Omega} g_{0}(\mathbf{u}) d \mathbf{x}
$$

Copyright $@$ by SIAM. Unauthorized reproduction of this article is prohibited. 
subject to (2.9). In fact, by the method of Lagrange multipliers, we define the Lagrangian function as

$$
L\left(u_{2}, \ldots, u_{N}, \theta_{2}, \ldots, \theta_{N}\right)=\int_{\Omega}\left(g_{0}(\mathbf{u})+\sum_{i=2}^{N} \theta_{i} u_{i}\right) d \mathbf{x}-\sum_{i=2}^{N} \theta_{i} u_{i}^{t},
$$

where $\theta_{i}(2 \leq i \leq N)$ are the Lagrange multipliers. Let $\left(u_{2}, \ldots, u_{N}\right)$ be a minimizer of the constrained problem, and then the necessary condition is that there exist $\theta_{i}$ such that

$$
\sum_{j=1}^{N} \mu_{j}^{0} \frac{\partial n_{j}}{\partial u_{i}}+\theta_{i}=0, \quad i=2, \ldots, N
$$

It is obtained by applying the relations given by $(2.5)-(2.7)$ to $(2.27)$ that

$$
\frac{\sqrt{c_{i}}}{\sqrt{\lambda}} \mu_{1}^{0}-\frac{\sqrt{c_{1}}}{\sqrt{\lambda}} \mu_{i}^{0}+\theta_{i}=0, \quad i=2, \ldots, N .
$$

Since (2.28) should hold for the bulk equilibrium phases, we get

$$
\theta_{i}=\frac{\sqrt{c_{1}}}{\sqrt{\lambda}} \mu_{i}-\frac{\sqrt{c_{i}}}{\sqrt{\lambda}} \mu_{1}
$$

Thus, (2.26) is reduced to

$$
L\left(u_{2}, \ldots, u_{N}\right)=\int_{\Omega}\left(g_{0}(\mathbf{u})-\boldsymbol{\mu}^{T} \mathbf{Q u}\right) d \mathbf{x}+\boldsymbol{\mu}^{T} \mathbf{Q} \mathbf{u}^{t} .
$$

The required result is obtained from Condition (A).

TheOrem 2.1. Under Condition (A), there exists $\mathbf{n} \in V^{C}$ such that (2.1) and (2.2) hold.

Proof. By the relation between $\mathbf{u}$ and $\mathbf{n}$, it suffices to prove the existence of $\mathbf{u}$. As an analogue of $V^{C}$, we define $V_{\mathbf{u}}^{C}$ as

$$
V_{\mathbf{u}}^{C}=\left\{\mathbf{u} \in\left(H^{1}(\Omega)\right)^{N}: \quad \int_{\Omega} \mathbf{u} d \mathbf{x}=\mathbf{u}^{t}\right\} .
$$

Choose a minimizing sequence $\left\{u^{k}\right\}_{k=1}^{\infty}$ in $H^{1}(\Omega)$ satisfying $u^{G} \leq u^{k} \leq u^{L}$ and the constraint (2.9). Condition (A) allows us to calculate the corresponding $\left\{u_{i}^{k}\right\}_{k=1}^{\infty}, i=$ $2, \ldots, N$, being the minimizers of the constrained minimization problem $(2.25)$ with the constraint (2.9). It is claimed that $G\left(\mathbf{u}^{k}\right)$ is bounded below since $g_{0}\left(\mathbf{u}^{k}\right)=$ $f_{0}\left(\mathbf{n}^{k}\right) \geq 0$ and $\int_{\Omega} \frac{1}{2} \lambda\left|\nabla u^{k}\right|^{2} d \mathbf{x} \geq 0$. Then we extract a subsequence, which is yet denoted by $\left\{\mathbf{u}^{k}\right\}_{k=1}^{\infty}$, such that

$$
G\left(\mathbf{u}^{k}\right) \rightarrow G_{0}=\inf _{\mathbf{u} \in V_{\mathbf{u}}^{C}} G(\mathbf{u})
$$

For a given small positive constant $\delta$, we have

$$
\int_{\Omega}\left(g_{0}\left(\mathbf{u}^{k}\right)+\frac{1}{2} \lambda\left|\nabla u^{k}\right|^{2}\right) d \mathbf{x} \leq G_{0}+\delta
$$

if only $k$ is sufficiently large. It is estimated with the expression of $f_{0}$ that

$$
\int_{\Omega} g_{0}\left(\mathbf{u}^{k}\right) d \mathbf{x} \leq C .
$$

Copyright $@$ by SIAM. Unauthorized reproduction of this article is prohibited. 
Consequently, we deduce from (2.32) and (2.33) that

$$
\int_{\Omega} \frac{1}{2} \lambda\left|\nabla u^{k}\right|^{2} d \mathbf{x} \leq C
$$

We then can extract a subsequence $\left\{u^{k_{j}}\right\}$ such that

$$
u^{k_{j}} \rightarrow u \text { weakly in } H^{1}(\Omega), u^{k_{j}} \rightarrow u \text { strongly in } L^{2}(\Omega) .
$$

It is easy to know that $u^{G} \leq u \leq u^{L}$. By Condition (A), there exist $u_{i}, i=2, \ldots, N$ minimizing $g_{0}$ under the constraint for $u$, and thus we have $G(\mathbf{u}) \leq G_{0}$.

The following theorem shows the relation between the surface tension functional and the Helmholtz free energy functional.

TheOREM 2.2. Under Condition (A), the minimizer of the surface tension $\sigma$ in $V^{C}$ exists and $\sigma$ is expressed as

$$
\sigma=\int_{\Omega}\left(\Phi(\mathbf{n})-\Phi_{B}+\frac{1}{2} \lambda|\nabla u|^{2}\right) d \mathbf{x},
$$

where $\mathbf{u}=\mathbf{Q}^{-1} \mathbf{n}$ and $\mathbf{n} \in V^{C}$ is the minimizer of (2.1) and (2.2).

Proof. From the definition of the grand potential $\Phi$ and the constraint (2.2), the surface tension is rewritten as

$$
\sigma(\mathbf{n})=F(\mathbf{n})-\sum_{i=1}^{N} \mu_{i} n_{i}^{t}-\Phi_{B}|\Omega|
$$

where $|\Omega|$ is the measure of $\Omega$. Thus, the existence of the minimizer for the surface tension $\sigma$ is obtained by the existence of the minimizer of the constrained problem (2.1) and (2.2) under Condition (A), and equation (2.36) results from the definition of $\mathbf{u}$.

2.4. Simplified Euler-Lagrange equations and maximum principle. In order to simulate the above interface model formulated with the constrained energy minimum, we need to solve the corresponding Euler-Lagrange equations, and moreover, by means of the Euler-Lagrange equations, we will prove the maximum principle of the weighted molar density, which plays an important role in both theory and numerical simulation. Assume that the boundary conditions are provided and the phase interfaces occur only in the interior of the domain $\Omega$. The minimizer of (2.1) constrained with (2.2), the equilibrium densities at the interface, must satisfy the following Euler-Lagrange equations [19, 25]

$$
\sum_{j=1}^{N} c_{i j} \Delta n_{j}=\mu_{i}^{0}-\mu_{i} \quad i=1, \ldots, N,
$$

where $\mu_{i}^{0}$ and $\mu_{i}$ are the chemical potentials. The boundary condition is given by

$$
\mathbf{n}=\mathbf{n}_{B} \text { at } \partial \Omega,
$$

where $\mathbf{n}_{B}=\mathbf{n}^{G}$ or $\mathbf{n}_{B}=\mathbf{n}^{L}$. The simplified Euler-Lagrange equations can be expressed as

$$
\begin{aligned}
\lambda \Delta u & =\sum_{i=1}^{N} \sqrt{c_{i} / \lambda}\left(\mu_{i}^{0}-\mu_{i}\right), \\
\sqrt{c_{1}}\left(\mu_{i}^{0}-\mu_{i}\right) & =\sqrt{c_{i}}\left(\mu_{1}^{0}-\mu_{1}\right), \quad 2 \leq i \leq N .
\end{aligned}
$$

Copyright (c) by SIAM. Unauthorized reproduction of this article is prohibited. 
Equations (2.39) and (2.40) are the multidimensional extension of the one-dimensional case derived in our previous work [20]. We note that (2.39) and (2.40) can also be deduced by applying the variation principle to the constrained minimum problem (2.8) with (2.9). To prove the maximum principle of weighted molar density in a multidimensional domain, we combine (2.40) and rewrite (2.39) to another simple form

$$
\sqrt{c_{1} \lambda} \Delta u=\mu_{1}^{0}-\mu_{1} .
$$

Theorem 2.3. Under Condition (A), the weighted density satisfies $u^{G} \leq u \leq u^{L}$ almost everywhere in $\Omega$.

Proof. To prove the maximum principle of $u$, we first extend the definitions of $\mu_{i}^{0}$ and define

$$
\bar{\mu}_{i}^{0}= \begin{cases}\mu_{i}^{0}, & u \in\left[u^{G}, u^{L}\right] \\ \mu_{i}, & u<u^{G} \text { or } u>u^{L} .\end{cases}
$$

Let $u^{-}=\min \left(u-u^{G}, 0\right)$ and then $u^{-} \leq 0$. It follows from (2.41) that

$$
\sqrt{c_{1} \lambda} \Delta\left(u-u^{G}\right)=\bar{\mu}_{1}^{0}-\mu_{1} .
$$

Multiplying both sides of (2.43) by $u^{-}$and integrating it over $\Omega$, with integration by parts, we get

$$
-\int_{\Omega} \sqrt{c_{1} \lambda} \nabla\left(u-u^{G}\right) \cdot \nabla u^{-} d \mathbf{x}=\int_{\Omega}\left(\bar{\mu}_{1}^{0}-\mu_{1}\right) u^{-} d \mathbf{x},
$$

where we have also used the fact $u^{-}=0$ on $\partial \Omega$. The right-hand side of (2.44) is estimated from (2.42) as

$$
\int_{\Omega}\left(\bar{\mu}_{1}^{0}-\mu_{1}\right) u^{-} d \mathbf{x}=0 .
$$

Thus, from (2.44) and (2.45), we find

$$
\int_{\Omega} \sqrt{c_{1} \lambda}\left|\nabla u^{-}\right|^{2} d \mathbf{x}=\int_{\Omega} \sqrt{c_{1} \lambda} \nabla\left(u-u^{G}\right) \cdot \nabla u^{-} d \mathbf{x}=0 .
$$

This implies that $u^{-}$is a constant almost everywhere in the domain $\Omega$, but the gas region is not empty, so we obtain $u^{-}=0$; that is, $u \geq u^{G}$ almost everywhere.

We turn to proving $u \leq u^{L}$. Let $u^{+}=\max \left(u-u^{L}, 0\right)$ and then $u^{+} \geq 0$. It is similar to obtain

$$
-\int_{\Omega} \sqrt{c_{1} \lambda}\left|\nabla u^{+}\right|^{2} d \mathbf{x}=\int_{\Omega}\left(\bar{\mu}_{1}^{0}-\mu_{1}\right) u^{+} d \mathbf{x}=0 .
$$

Finally, we conclude that $u \leq u^{L}$ almost everywhere.

3. Numerical methods. In practical applications, numerical simulation of the two-phase interface model is a main instrument for predicting the surface tension of multicomponent two-phase mixtures. In this section, we consider numerical methods for this problem. First, we note that the system of Euler-Lagrange equations is composed of one nonlinear partial differential equation and $N-1$ nonlinear algebraic equations. So we need to design both efficient nonlinear iterative methods and spatial discretization methods. 

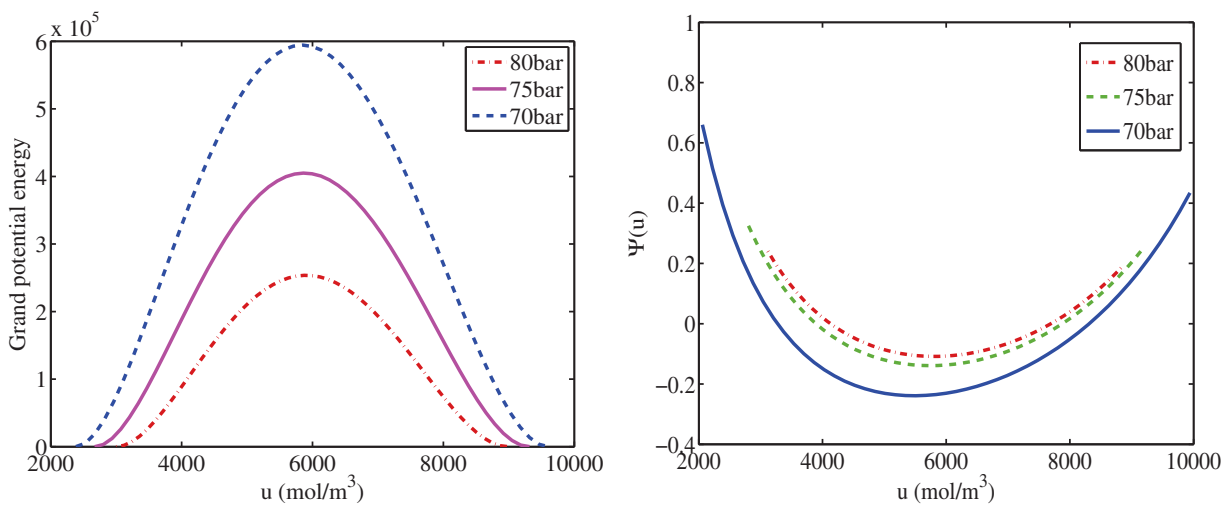

FiG. 1. Grand potential energy (left) and $\Psi(u)$ (right).

3.1. A modified Newton's method for Euler-Lagrange equations. It is well known that Newton's method is the most popular method to solve the related nonlinear equations $[15,34]$. However, the standard Newton's method solves a system of nonlinear equations in a simultaneous and coupled way, but this way may be not perfect for the considered nonlinear system because it consists of one nonlinear partial differential equation and $N-1$ nonlinear algebraic equations. In addition, Newton's method cannot ensure the maximum principle of the weighted molar density. The local minimum grand potential energy condition permits us to design a decoupled numerical method based on the classical Newton's method. From this, we now present a modified Newton's method with a relaxation factor for solving our problem.

Let $u^{k}$ be a given approximation of the exact weighted density solution, where the superscript $k$ represents the $k$ th Newton iteration. Suppose that $u^{G} \leq u^{k} \leq$ $u^{L}$; according to the local minimum grand potential energy condition, the minimizer $\left(u_{2}^{k}, \ldots, u_{N}^{k}\right)$ of $q\left(u_{2}, \ldots, u_{N} ; u^{k}\right)$ is the solution of the following nonlinear equations:

$$
\sqrt{c_{1}}\left(\mu_{i}^{0}\left(\mathbf{Q u}{ }^{k}\right)-\mu_{i}\right)=\sqrt{c_{i}}\left(\mu_{1}^{0}\left(\mathbf{Q u}{ }^{k}\right)-\mu_{1}\right), \quad 2 \leq i \leq N .
$$

The equations given by (3.1) can be solved by Newton's method, and then the mixture composition is calculated by $\mathbf{n}^{k}=\mathbf{Q} \mathbf{u}^{k}$. The new approximation $u^{k+1}$ of $u$ is obtained by solving

$$
-\lambda \Delta u^{k+1}+\left(\theta+\Psi\left(u^{k}\right)\right)\left(u^{k+1}-u^{k}\right)+\sqrt{\lambda / c_{1}}\left(\mu_{1}^{0}\left(\mathbf{n}^{k}\right)-\mu_{1}\right)=0,
$$

where $\theta \geq 0$ is a relaxation factor. In $(3.2), \Psi(u)$ is defined as

$$
\Psi(u)=\sum_{j=1}^{N} \sqrt{\lambda / c_{1}} \frac{\partial \mu_{1}^{0}(\mathbf{Q u})}{\partial u_{j}} \psi_{j}^{\prime}(u),
$$

where $u \in\left[u^{G}, u^{L}\right]$ and $u_{i}=\psi_{i}(u), i=2, \ldots, N$. In this method, the nonlinear algebraic equations (3.1) and the nonlinear partial differential equation (3.2) can be solved by a decoupled way.

There are two main reasons for using the relaxation factor in (3.2). One reason is that the relaxation factor is used to keep the maximum principle of the weighted density; the other is to guarantee the decay of approximate surface tensions with iterations. In addition, the properties of grand potential energy and its second derivative $\Psi(u)$ also have an effect on Newton's method. In Figure 1, we illustrate the profiles 
of the grand potential energy and its second derivative with respect to $u, \Psi(u)$, of a mixture composed of methane and pentane at the temperature $260 \mathrm{~K}$ and three different pressures (70bar, 75bar, and 80bar). It is observed that the grand potential is increasing as the weighted density $u$ goes away from the bulk equilibrium phases and it attains the maximum at one point $u^{*}$ that is near the middle point of the interval $\left[u^{G}, u^{L}\right]$, and correspondingly, $\Psi(u)$ is not always positive when $u$ varies from $u^{G}$ and $u^{L}$. So by using a suitable relaxation factor, we can keep the positivity of the operator $(-\lambda \Delta+\theta+\Psi(u))$.

The following theorem shows that the proposed modified Newton's method is capable of keeping the maximum principle of the weighted density such that we can always apply the local minimum grand potential energy condition.

THEOREM 3.1. Suppose that $u^{G} \leq u^{k} \leq u^{L}$; then there exists a suitable $\theta$ such that the new approximation $u^{k+1}$ produced by the modified Newton's method satisfies $u^{G} \leq u^{k+1} \leq u^{L}$ almost everywhere in $\Omega$.

Proof. Let $u_{-}^{k+1}=\min \left(u^{k+1}-u^{G}, 0\right)$. Multiplying both sides of (3.2) by $u_{-}^{k+1}$ and integrating it over $\Omega$, by using integration by parts, we get

$$
\begin{aligned}
\lambda \| & \nabla u_{-}^{k+1} \|_{L^{2}}^{2}+\int_{\Omega}\left(\theta+\Psi\left(u^{k}\right)\right)\left(u_{-}^{k+1}\right)^{2} d \mathbf{x} \\
= & \int_{\Omega}\left(\theta+\Psi\left(u^{k}\right)\right)\left(u^{k}-u^{G}\right) u_{-}^{k+1} d \mathbf{x}+\int_{\Omega} \sqrt{\lambda / c_{1}}\left(\mu_{1}-\mu_{1}^{0}\left(\mathbf{n}^{k}\right)\right) u_{-}^{k+1} d \mathbf{x},
\end{aligned}
$$

where we have also used the fact $u_{-}^{k+1}=0$ on $\partial \Omega$. If $\left\|u_{-}^{k+1}\right\|_{L^{2}}=0$, then $u^{k+1} \geq$ $u^{G}$ almost everywhere in $\Omega$; otherwise, we have $\left\|u_{-}^{k+1}\right\|_{L^{2}}>0$. Denote $\Psi_{M}=$ $\max _{u \in\left[u^{G}, u^{L}\right]}|\Psi(u)|$, and choose $\theta \geq 2 \Psi_{M}$. The second term of the left-hand side in (3.4) is bounded below as

$$
\int_{\Omega}\left(\theta+\Psi\left(u^{k}\right)\right)\left(u_{-}^{k+1}\right)^{2} d \mathbf{x} \geq\left(\theta-\Psi_{M}\right)\left\|u_{-}^{k+1}\right\|_{L^{2}}^{2} \geq \Psi_{M}\left\|u_{-}^{k+1}\right\|_{L^{2}}^{2} .
$$

The two terms of the right-hand side in (3.4) are estimated as

$$
\begin{aligned}
& \int_{\Omega}\left(\theta+\Psi\left(u^{k}\right)\right)\left(u^{k}-u^{G}\right) u_{-}^{k+1} d \mathbf{x}+\int_{\Omega} \sqrt{\lambda / c_{1}}\left(\mu_{1}-\mu_{1}^{0}\left(\mathbf{n}^{k}\right)\right) u_{-}^{k+1} d \mathbf{x} \\
& \quad=\int_{\Omega}\left(\theta+\Psi\left(u^{k}\right)-\Psi\left(u_{\xi}^{k}\right)\right)\left(u^{k}-u^{G}\right) u_{-}^{k+1} d \mathbf{x} \\
& \quad \leq 0,
\end{aligned}
$$

where we have used the following fact:

$$
\sqrt{\lambda / c_{1}}\left(\mu_{1}^{0}\left(\mathbf{n}^{k}\right)-\mu_{1}\right)=\Psi\left(u_{\xi}\right)\left(u^{k}-u^{G}\right), \quad u_{\xi}^{k} \in\left[u^{G}, u^{k}\right] .
$$

It is obtained from the above estimates that $\left\|u_{-}^{k+1}\right\|_{L^{2}} \leq 0$, and thus $u^{k+1} \geq u^{G}$ almost everywhere in $\Omega$. Let $u_{+}^{k+1}=\max \left(u^{k+1}-u^{L}, 0\right)$; then we can also prove $u^{k+1} \leq u^{L}$ by the similar routine.

The following theorem shows that the proposed modified Newton's method can guarantee the decay of approximate surface tensions with iterations.

Theorem 3.2. Suppose that $u^{G} \leq u^{k} \leq u^{L}$; then there exists a suitable $\theta$ such that

$$
\frac{1}{2} \lambda\left\|\nabla u^{k+1}\right\|_{L^{2}}^{2}+\left(\Phi\left(\mathbf{n}^{k+1}\right)-\Phi_{B}, 1\right) \leq \frac{1}{2} \lambda\left\|\nabla u^{k}\right\|_{L^{2}}^{2}+\left(\Phi\left(\mathbf{n}^{k}\right)-\Phi_{B}, 1\right) .
$$

Copyright (c) by SIAM. Unauthorized reproduction of this article is prohibited. 
Proof. First, from Theorem 3.1, we choose $\theta \geq 2 \Psi_{M}$, where $\Psi_{M}=\max _{u \in\left[u^{G}, u^{L}\right]}$ $|\Psi(u)|$, so that $u^{G} \leq u^{k+1} \leq u^{L}$ almost everywhere in $\Omega$. Multiplying (3.2) by $\left(u^{k+1}-u^{k}\right)$ and integrating it over $\Omega$, we obtain

$$
\begin{gathered}
\frac{1}{2} \lambda\left(\left\|\nabla u^{k+1}\right\|_{L^{2}}^{2}-\left\|\nabla u^{k}\right\|_{L^{2}}^{2}\right)+\frac{1}{2} \lambda\left\|\nabla\left(u^{k+1}-u^{k}\right)\right\|_{L^{2}}^{2}+\theta\left\|u^{k+1}-u^{k}\right\|_{L^{2}}^{2} \\
(3.9) \quad+\sqrt{\lambda / c_{1}}\left(\mu_{1}^{0}\left(\mathbf{n}^{k}\right)-\mu_{1}, u^{k+1}-u^{k}\right)+\left(\Psi\left(u^{k}\right)\left(u^{k+1}-u^{k}\right), u^{k+1}-u^{k}\right)=0 .
\end{gathered}
$$

With the help of the linear transformation, we find

$$
\begin{aligned}
& \sqrt{\lambda / c_{1}}\left(\mu_{1}^{0}\left(\mathbf{n}^{k}\right)-\mu_{1}, u^{k+1}-u^{k}\right) \\
& =\sum_{i=1}^{N} \sqrt{c_{i} / \lambda}\left(\mu_{i}^{0}\left(\mathbf{n}^{k}\right)-\mu_{i}, u^{k+1}-u^{k}\right) \\
& =\left(\sum_{i=1}^{N} \sqrt{c_{i} / \lambda}\left(\mu_{i}^{0}\left(\mathbf{n}^{k}\right)-\mu_{i}\right), \sum_{j=1}^{N} \sqrt{c_{j} / \lambda}\left(n_{j}^{k+1}-n_{j}^{k}\right)\right) \\
& =\frac{1}{\lambda} \sum_{i=1}^{N} \sum_{j=1}^{N} \sqrt{c_{i} c_{j}}\left(\mu_{i}^{0}\left(\mathbf{n}^{k}\right)-\mu_{i}, n_{j}^{k+1}-n_{j}^{k}\right) \\
& =\frac{1}{\lambda} \sum_{i=1}^{N} \sum_{j=1}^{N} c_{i}\left(\mu_{j}^{0}\left(\mathbf{n}^{k}\right)-\mu_{j}, n_{j}^{k+1}-n_{j}^{k}\right) \\
& =\sum_{j=1}^{N}\left(\mu_{j}^{0}\left(\mathbf{n}^{k}\right)-\mu_{j}, n_{j}^{k+1}-n_{j}^{k}\right) .
\end{aligned}
$$

It follows from Taylor's theorem that

$$
\begin{aligned}
\left(\Phi\left(\mathbf{n}^{k+1}\right)-\Phi\left(\mathbf{n}^{k}\right), 1\right)= & \sum_{j=1}^{N}\left(\mu_{j}^{0}\left(\mathbf{n}^{k}\right)-\mu_{j}, n_{j}^{k+1}-n_{j}^{k}\right) \\
& +\frac{1}{2} \sum_{i=1}^{N} \sum_{j=1}^{N}\left(\frac{\partial \mu_{j}^{0}\left(\mathbf{n}_{*}\right)}{\partial n_{i}}\left(n_{i}^{k+1}-n_{i}^{k}\right), n_{j}^{k+1}-n_{j}^{k}\right) \\
= & \sqrt{\lambda / c_{1}}\left(\mu_{1}^{0}\left(\mathbf{n}^{k}\right)-\mu_{1}, u^{k+1}-u^{k}\right) \\
& +\frac{1}{2}\left(\Psi\left(u_{*}\right)\left(u^{k+1}-u^{k}\right),\left(u^{k+1}-u^{k}\right)\right),
\end{aligned}
$$

where $\mathbf{n}_{*}$ is between $\mathbf{n}^{k}$ and $\mathbf{n}^{k+1}$ and $u_{*} \in\left[u^{G}, u^{L}\right]$. Thus,

$$
\begin{gathered}
\sqrt{\lambda / c_{1}}\left(\mu_{1}^{0}\left(\mathbf{n}^{k}\right)-\mu_{1}, u^{k+1}-u^{k}\right)+\left(\Psi\left(u^{k}\right)\left(u^{k+1}-u^{k}\right), u^{k+1}-u^{k}\right) \\
(3.12)=\left(\Phi\left(\mathbf{n}^{k+1}\right)-\Phi\left(\mathbf{n}^{k}\right), 1\right)+\left(\left(\Psi\left(u^{k}\right)-\frac{1}{2} \Psi\left(u_{*}\right)\right)\left(u^{k+1}-u^{k}\right), u^{k+1}-u^{k}\right) .
\end{gathered}
$$

It follows from the combination of(3.9) and (3.12) that

$$
\begin{aligned}
\frac{1}{2} \lambda\left(\left\|\nabla u^{k+1}\right\|_{L^{2}}^{2}-\left\|\nabla u^{k}\right\|_{L^{2}}^{2}\right)+ & \frac{1}{2} \lambda\left\|\nabla\left(u^{k+1}-u^{k}\right)\right\|_{L^{2}}^{2}+\theta\left\|u^{k+1}-u^{k}\right\|_{L^{2}}^{2} \\
& +\left(\Phi\left(\mathbf{n}^{k+1}\right)-\Phi\left(\mathbf{n}^{k}\right), 1\right) \leq \frac{3}{2} \Psi_{M}\left\|u^{k+1}-u^{k}\right\|_{L^{2}}^{2} .
\end{aligned}
$$

Copyright $@$ by SIAM. Unauthorized reproduction of this article is prohibited. 
Since we have chosen the relaxation factor $\theta \geq 2 \Psi_{M}$, the required inequality is obtained from (3.13).

The maximum principle has a very important effect on the robustness of the proposed Newton's method, so in order to keep this key property, we need to design an appropriate choice of relaxation factor. We first calculate approximately $\theta_{J}=$ $2 \max _{u \in\left[u^{G}, u^{L}\right]}|\Psi(u)|$. The proof of Theorem 3.1 indicates that the maximum principle can be reached if $\theta \geq \theta_{J}$. On the other hand, from our numerical tests, we observe that actally in some iterations, the relaxation factor must be chosen greater than zero to keep the maximum principle, but it is also satisfied in many other cases when $\theta=0$. So in these cases, the choice of $\theta=0$ may speed up the convergence of Newton's method because Newton's method has locally quadratical convergence for $\theta=0$. Based on the above analysis, we propose the following adaptive strategy for choosing the relaxation factor in practical computation. For each Newton iteration, the initial value of the relaxation factor is taken as $\theta=0$, and then one solves (3.2) to get $u^{k+1}$ and check whether the maximum principle is reached. If so, one accepts it and continues the next Newton iterative step; otherwise, one can take $\theta=\theta_{J}$ to repeat the above computational procedure. If the maximum principle is not yet reached when $\theta=\theta_{J}$, we use a cutoff operator as $u^{0}=\min \left(\max \left(u^{k+1}, u^{G}\right), u^{L}\right)$ to restart Newton iteration. We also note that the cutoff operator is mainly used to deal with the cases in which the maximum principle is disturbed by the error caused from the computational precision. It is observed from our numerical tests that this adaptive choice strategy for the relaxation factor can well guarantee the convergence of Newton's method, and moreover, the very large relaxation factor is also avoided.

3.2. Adaptive finite element approximations. We now consider the adaptive finite element method that will be used as a spatial discretization of our problem. Let $V_{h} \subset V$ be composed of continous piecewise polynomials. Find a $\mathbf{n}_{h} \in V_{h}$ satisfying

$$
F\left(\mathbf{n}_{h}\right)=\min _{\widetilde{\mathbf{n}}_{h} \in V_{h}} F\left(\widetilde{\mathbf{n}}_{h}\right)
$$

subject to $\int_{\Omega} \mathbf{n}_{h} d \mathbf{x}=\mathbf{n}^{t}$. It can be proved that $\mathbf{n} \in\left(H^{2}(\Omega)\right)^{N}$, but $\mathbf{n}$ may have higher-order regularity if $f_{0}$ and $\psi_{i}$ are sufficiently smooth, so we assume $\mathbf{n} \in$ $\left(H^{s+1}(\Omega)\right)^{N}, s \geq 1$, in the following finite element analysis. The finite element approximation of surface tension is

$$
\sigma_{h}=\int_{\Omega}\left(\Phi\left(\mathbf{n}_{h}\right)-\Phi_{B}+\frac{1}{2} \lambda\left|\nabla u_{h}\right|^{2}\right) d \mathbf{x}
$$

where $\mathbf{u}_{h}=\mathbf{Q}^{-1} \mathbf{n}_{h}$.

Theorem 3.3. Let $r \geq 1$ be the polynomial degree. Under Condition (A), then $F\left(\mathbf{n}_{h}\right)$ converges to $F(\mathbf{n})$, and thus $\sigma_{h}$ also converges to $\sigma$ as the mesh size $h \rightarrow 0$. Moreover, we have the following error estimate:

$$
0 \leq \sigma_{h}-\sigma \leq C h^{\min (r, s)}\|\mathbf{n}\|_{\left(H^{s+1}(\Omega)\right)^{N}} .
$$

Proof. Let $\widehat{\mathbf{n}}$ be the interpolation approximations of $\mathbf{n}$ in $V_{h}$ and let $\widehat{\sigma}=\sigma(\widehat{\mathbf{n}})$. By using the linear transformation, we get $\widehat{\mathbf{u}}=\mathbf{Q}^{-1} \widehat{\mathbf{n}}$. Since $\widehat{\mathbf{n}}_{h}$ converges to $\mathbf{n}$ in $V$ as $h \rightarrow 0$, for some positive number $\alpha$, we have $F\left(\widehat{\mathbf{n}}_{h}\right) \leq F(\mathbf{n})+\alpha$ if $h$ is sufficiently small. Take a minimizing sequence $\left\{\mathbf{n}_{h}^{k}\right\}_{k=1}^{\infty} \in V_{h}$ such that

$$
G\left(\mathbf{u}_{h}^{k}\right)=F\left(\mathbf{n}_{h}^{k}\right) \rightarrow F_{0}^{h} .
$$


By similar techniques used in the proof of Theorem 2.1, we can derive the existence of the finite element approximation of $\mathbf{u}_{h}$ and $\mathbf{n}_{h}$. Since $\mathbf{n}_{h}$ is the minimizer of $F$ in $V_{h}$, it holds that $G\left(\mathbf{u}_{h}\right)=F\left(\mathbf{n}_{h}\right) \leq F\left(\widehat{\mathbf{n}}_{h}\right) \leq F(\mathbf{n})+\alpha$; that is,

$$
F(\mathbf{n}) \leq \int_{\Omega}\left(g_{0}\left(\mathbf{u}_{h}\right)+\frac{1}{2} \lambda\left|\nabla u_{h}\right|^{2}\right) d \mathbf{x}=G\left(\mathbf{u}_{h}\right)=F\left(\mathbf{n}_{h}\right) \leq F(\mathbf{n})+\alpha .
$$

As a result, $F\left(\mathbf{n}_{h}\right)$ converges to $F(\mathbf{n})$ as $h \rightarrow 0$; from the relation between the surface tension functional and the Helmholtz free energy functional, we also obtain that $\sigma_{h}$ converges to $\sigma$ as $h \rightarrow 0$. It is known that $\sigma \leq \widehat{\sigma}$ since the finite element space is a subspace of $V$. With the approximation properties, we have

$$
\begin{aligned}
\widehat{\sigma}-\sigma & =\int_{\Omega}\left(g_{0}(\widehat{\mathbf{u}})-g_{0}(\mathbf{u})-\boldsymbol{\mu}^{T} \mathbf{Q}(\widehat{\mathbf{u}}-\mathbf{u})+\frac{1}{2} \lambda\left(|\nabla \widehat{u}|^{2}-|\nabla u|^{2}\right)\right) d \mathbf{x} \\
& \leq C\|\widehat{\mathbf{u}}-\mathbf{u}\|_{L^{2}(\Omega)}+\frac{1}{2} \lambda\left(\|\nabla(\widehat{u}-u)\|_{L^{2}(\Omega)}+2\|\nabla u\|_{L^{2}(\Omega)}\right)\|\nabla(\widehat{u}-u)\|_{L^{2}(\Omega)} \\
& \leq C h^{\min (r, s)}\|\mathbf{n}\|_{H^{s+1}(\Omega)} .
\end{aligned}
$$

The finite element approximations of the surface tension has the error estimate

$$
0 \leq \sigma_{h}-\sigma \leq \widehat{\sigma}-\sigma \leq C h^{\min (r, s)}\|\mathbf{n}\|_{H^{s+1}(\Omega)},
$$

which also implies that $\sigma_{h}$ converges to $\sigma$ as $h \rightarrow 0$.

Adaptive finite element methods have been a fundamental numerical instrument for solving the partial differential equations in scientific and engineering problems $[2,7,23,38,39,41]$. Adaptive finite element methods have been also successfully used in [19] for the surface tension computation since the adaptive technique is capable of effectively capturing the location of interface between two bulk phases. Similar to [19], we now introduce a physical-based mesh-adaptive method. Let $\mathcal{E}_{h}$ denote a nondegenerate mesh partition of the domain $\Omega$. The discrete surface tension on each element $E$ is defined by

$$
\eta_{h}(E)=\int_{E}\left(\Phi\left(\mathbf{n}_{h}\right)-\Phi_{B}+\frac{1}{2} \lambda\left|\nabla u_{h}\right|^{2}\right) d \mathbf{x}
$$

Then the discrete surface tension on the mesh $\mathcal{E}_{h}$ is written as $\sigma_{h}=\eta_{h}\left(\mathcal{E}_{h}\right)=$ $\sum_{E \in \mathcal{E}_{h}} \eta_{h}(E)$. We first choose an initial uniform mesh $\mathcal{E}_{h_{0}}$ with mesh size $h_{0}$, and compute finite element solutions $u_{h_{0}}$ and $\mathbf{n}_{h_{0}}$ by using the proposed Newton's method. For given solutions $u_{h_{k}}$ and $\mathbf{n}_{h_{k}}(k=0,1,2, \ldots)$ computed on the mesh $\mathcal{E}_{h_{k}}$ with grid size $h_{k}$, we calculate $\eta_{h}(E)$ with $\mathcal{E}_{h}=\mathcal{E}_{h_{k}}$ in (3.21). The mesh-refinement procedure stops if the following stopping criterion is satisfied:

$$
\sigma_{h_{k}}-\sigma_{h_{k+1}} \leq \epsilon,
$$

where $\epsilon>0$ is a given tolerance. If (3.22) is violated, we construct a new mesh $\mathcal{E}_{h_{k+1}}$ with the size $h_{k+1}$ by refining the elements satisfying $\eta_{h_{k}}(E) \geq \chi_{k}$, where $0<\chi_{k}<\sigma_{h_{k}}$. From Theorem 3.3, we have the following estimate:

$$
0 \leq \sigma_{h_{k}}-\sigma_{h_{k+1}} \leq \sigma_{h_{k}}-\sigma \leq \sigma_{h_{k}}-\sigma_{h_{k+1}}+C h_{k+1}^{\gamma},
$$

where $\gamma=\min (r, s)$. In this adaptive method, we refine the elements with large 
contributions to the surface tension, and as a result, the error between the exact and approximate surface tensions is reduced, and moreover, approximate surface tensions are monotone decreasing with the local mesh refinement, which is in agreement with the minimum property of surface tension. Since the refinement indicator is based on the physical property of surface tension, it is a physical-based adaptive meshrefinement algorithm in practical applications.

4. Numerical tests. We now simulate multicomponent two-phase hydrocarbon fluid interface problems. These numerical tests are carried out for the two main purposes: the first one is to verify the local minimum grand potential energy condition, and the second one is to show the performance of the adaptive finite element method with the modified Newton's method. The tested mixtures are composed of binary hydrocarbons and terry hydrocarbons, respectively. The PR-EOS [28] is used to compute the equilibrium properties of the bulk phases, such as the molar densities and the chemical potentials; the detailed formulation of PR-EOS can be found in the appendix.

In the tested one-dimensional problems, the length of the one-dimensional domain is taken equal to $10 \mathrm{~nm}$. The initial mesh is uniform with 20 elements, and for the initial mesh, the initial approximation $u^{0}$ of Newton's method is taken as $u^{0}=u^{G}$ in the first ten elements and $u^{0}=u^{L}$ in the last ten elements. For the boundary conditions, we take the bulk gas phase as the left-hand end, and the right-hand end is the bulk liquid phase.

For two-dimensional problems, the tested domain is an annular region located between an interior circle with the radius $3 \mathrm{~nm}$ and an exterior circle with the radius $10 \mathrm{~nm}$. For the boundary conditions, we impose $\mathbf{n}=\mathbf{n}^{G}$ on the exterior circle boundary and $\mathbf{n}=\mathbf{n}^{L}$ on the interior circle boundary. The initial approximation $\mathbf{n}^{0}$ of Newton's method is taken as $\mathbf{n}^{0}=\mathbf{n}^{G}$ in the entire domain.

The linear adaptive finite element method is used for all examples. To obtain the accurate values of surface tension and mixture compositions, the mesh is adaptively refined twice during the computations, and in every mesh refinement, the refined elements with large surface tension contributions account for about $30-40 \%$ of all elements. On each refined mesh, the initial approximation of Newton's method is calculated by the interpolation of the solutions on the coarse mesh.

4.1. Binary mixture. The tested binary mixture is composed of methane $\left(\mathrm{C}_{1}\right)$ and pentane $\left(\mathrm{C}_{5}\right)$. Figure 2 illustrates the grand potential energy profiles for three different weighted molar densities (i.e., $u=5999 \mathrm{~mol} / \mathrm{m}^{3}, 7313 \mathrm{~mol} / \mathrm{m}^{3}$, and $9119 \mathrm{~mol} / \mathrm{m}^{3}$, respectively) of the binary mixture composed of $\mathrm{C}_{1}$ and $\mathrm{C}_{5}$ at $260 \mathrm{~K}$ and two different pressures (60bar and 70bar). It is observed that for any given weighted molar density, the grand potential energy can be viewed as a convex function of $u_{2}$, and as a result, there exists a unique minimizer such that the grand potential energy function attains its minimum. Figures 3 and 4 show more profiles of the grand potential energy for given weighted molar densities at different temperatures and pressures, which also verify the local minimum grand potential energy condition. We must note that from Figure 5, the grand potential energy function is not convex with respect to the two variables $u$ and $u_{2}$, so in this case, it attains its minimum only in the bulk liquid and gas phases. Thus, the local minimum grand potential energy condition is an interesting and important property, and in all numerical tests of this paper, this condition is always used for effectively calculating the mixture compositions for given weighted molar density. 

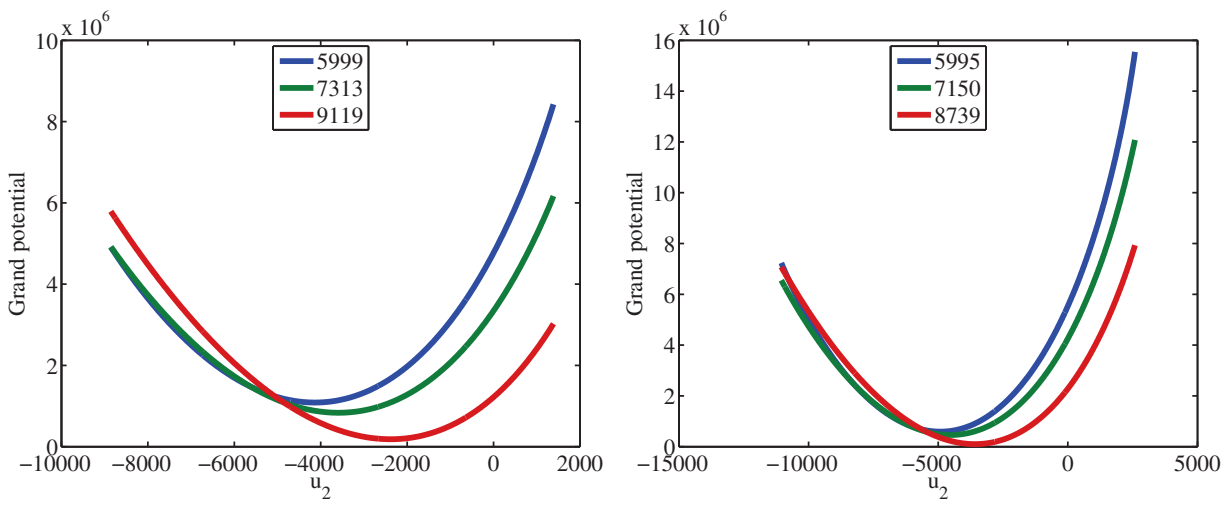

FIG. 2. Grand potential energy profiles of the binary mixture with different weighted molar densities at $260 \mathrm{~K}$ and different pressures: 60bar (left), 70bar (right).
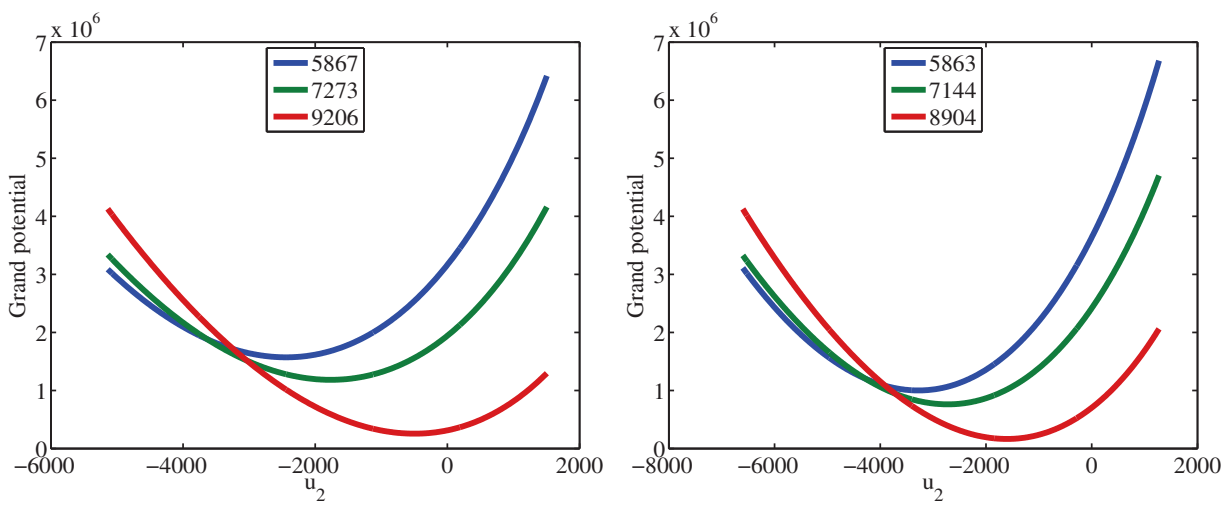

FIG. 3. Grand potential energy profiles of the binary mixture with different weighted molar densities at $270 \mathrm{~K}$ and different pressures: 50bar (left), 60bar (right).
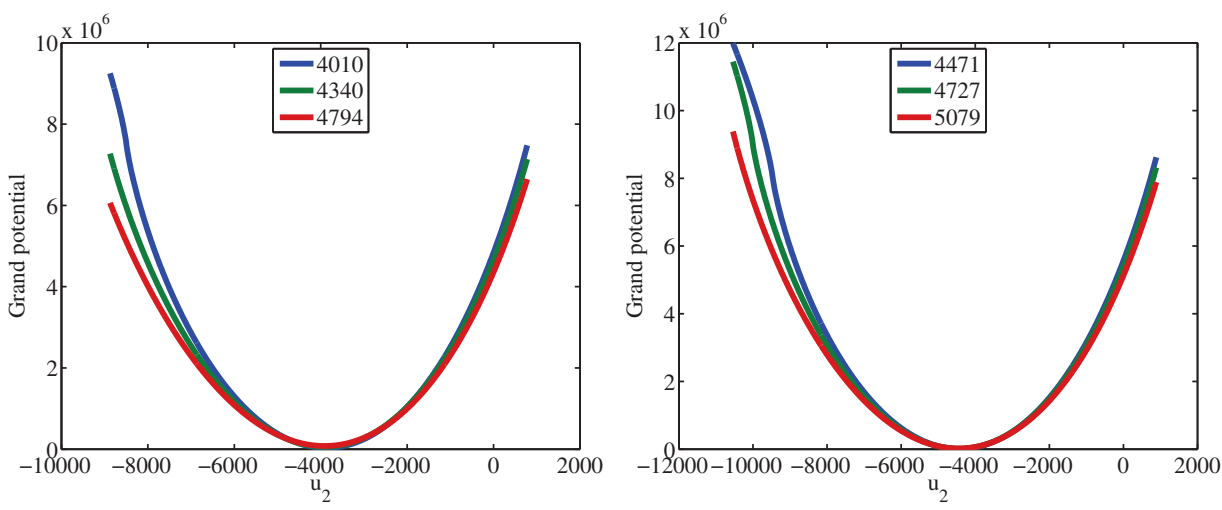

FIG. 4. Grand potential energy profiles of the binary mixture with different weighted molar densities at $280 \mathrm{~K}$ and different pressures: 80bar (left), 90bar (right).

Using the adaptive finite element method with the modified Newton's method, we calculate the surface tension for the tested binary mixture at the temperature $260 \mathrm{~K}$ and different pressures, which are illustrated in Figure 6. It is observed that the cal- 

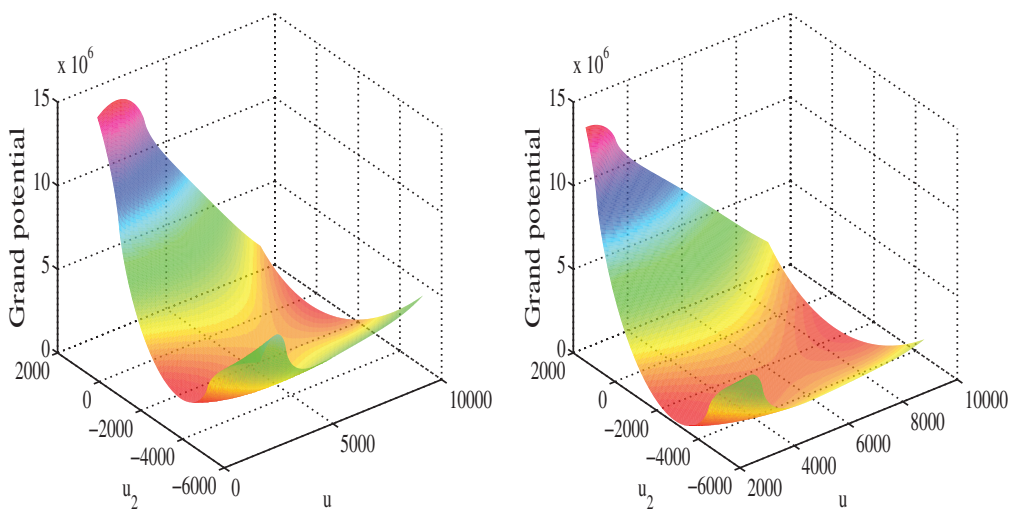

FIG. 5. Grand potential energy profiles of the binary mixture at $270 \mathrm{~K}$ and different pressures: 50 bar (left), 60 bar (right).

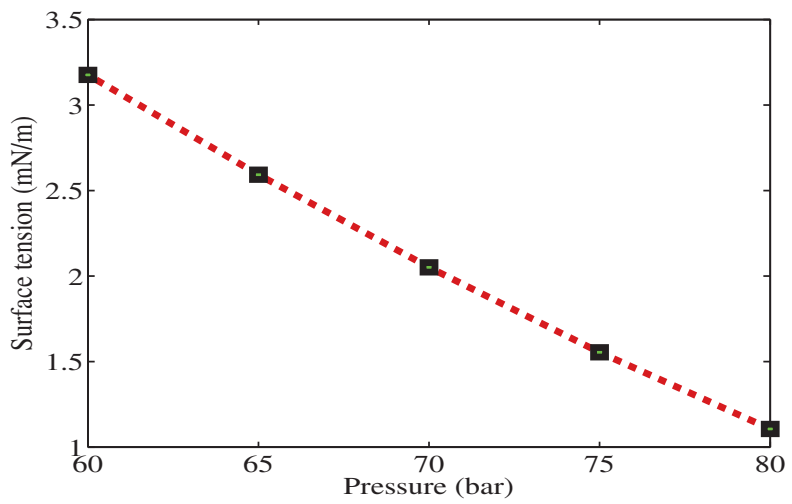

FIG. 6. Surface tension of the binary mixture at $260 \mathrm{~K}$ and different pressures.

culated surface tensions drop as the pressure is increasing in a range. Figure 7 depicts the calculated molar density profiles at $260 \mathrm{~K}$ and different pressures, which show the nonmonotonicity distributions of methane within the two-phase interfaces. Figure 8 depicts the calculated weighted molar density profiles at $260 \mathrm{~K}$ and different pressures, which show its monotonicity distributions within the two-phase fluid interfaces. Figure 8 also shows the distributions of the plotting points, and from these figures, we see that the adaptive method is capable of refining adaptively and accurately the regions in which the weighted molar density varies fast, and thus the total number of the required elements to attain high accuracy is less than that of the uniform mesh.

For two-dimensional cases, we simulate this binary mixture at the temperature $260 \mathrm{~K}$ and the pressure 70bar. The total surface tensions calculated on the initial uniform mesh and refined meshes are shown in Figure 9. It is observed that the total surface tension is evidently decreased with the local mesh refinement. The initial uniform mesh and two refined meshes are illustrated in Figure 10. In Figure 11, we illustrate the molar density of each component and the weighted molar density computed on the twice refined mesh. From these results, we can see that the local mesh refinement can also match the mixture composition distribution; that is, the locally refined elements are located in the regions with the large variation of weighted molar density. 

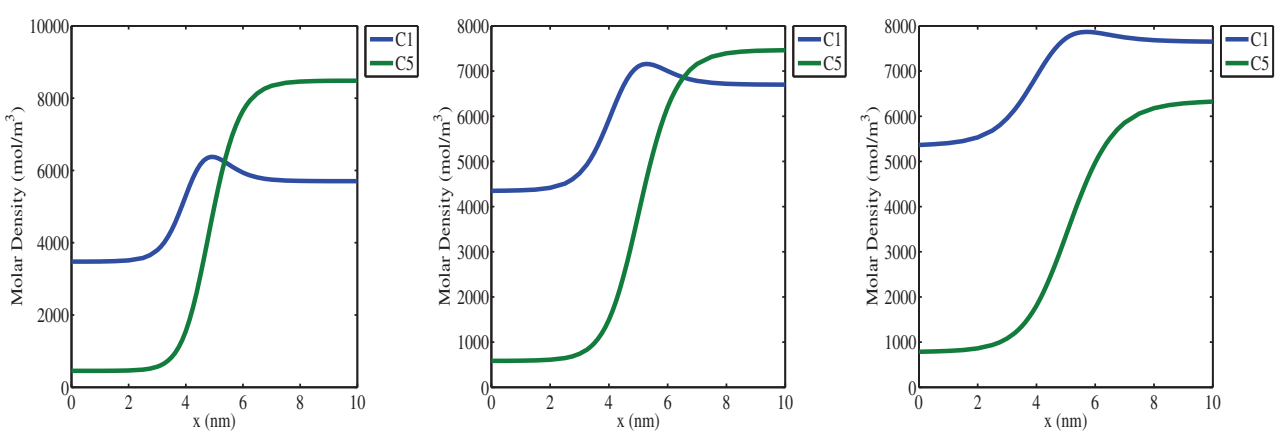

FIG. 7. Binary mixture molar density profiles at $260 \mathrm{~K}$ and different pressures: 60bar (left), 70 bar (center), 80bar (right).
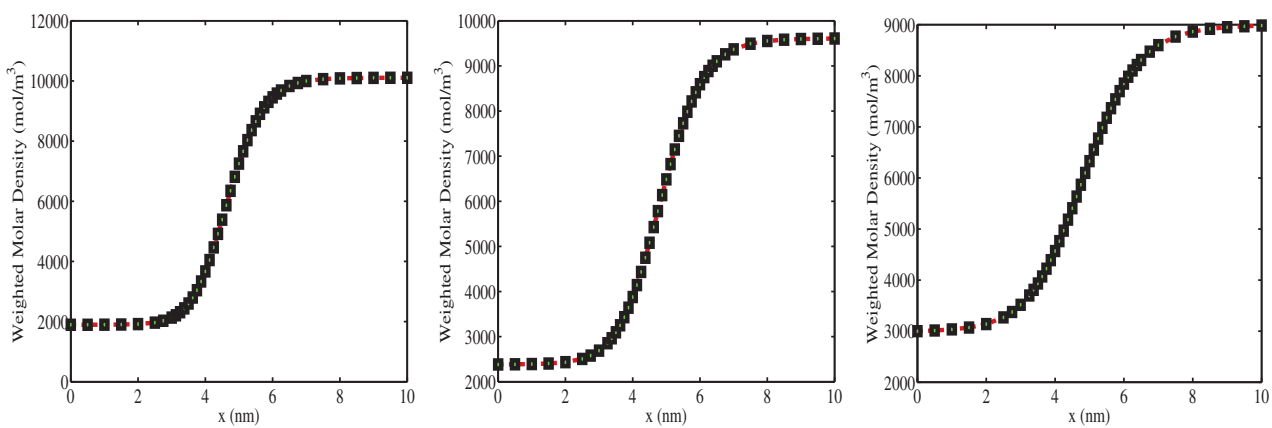

FIG. 8. Binary mixture weighted molar density profiles at $260 \mathrm{~K}$ and different pressures: 60bar (left), 70bar (center), 80bar (right).

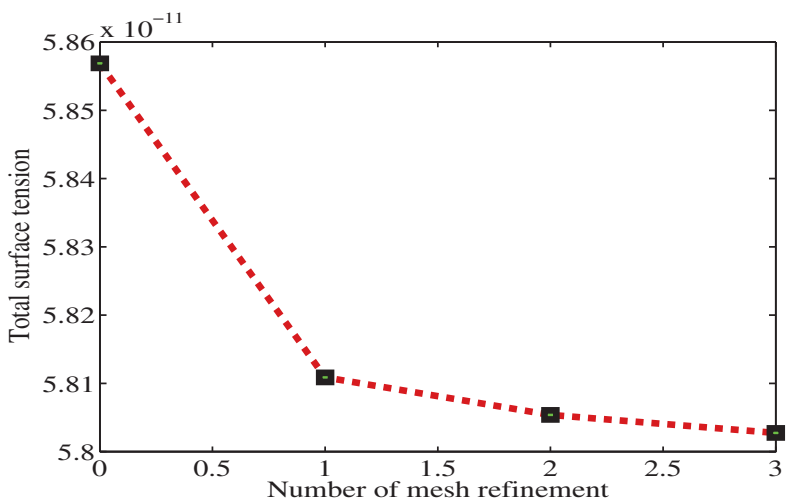

FIG. 9. Total surface tensions of the binary mixture in the two-dimensional problem at $260 \mathrm{~K}$ and $70 b a r$.

4.2. Ternary mixture. The ternary mixture is composed of $80 \%$ methane $\left(\mathrm{C}_{1}\right)$, $15 \%$ propane $\left(\mathrm{C}_{3}\right)$, and $5 \%$ decane $\left(\mathrm{C}_{10}\right)$ at the temperature $300 \mathrm{~K}$ and different pressures. Figures 12-14 illustrate the contour surfaces of the grand potential energy function of two variables $u_{2}$ and $u_{3}$ for various given values of the weighted molar density $(u)$ at the temperature $300 \mathrm{~K}$ and different pressures. In these figures, we also mark by the red dots the approximate minimum points of the grand potential

Copyright (c) by SIAM. Unauthorized reproduction of this article is prohibited. 

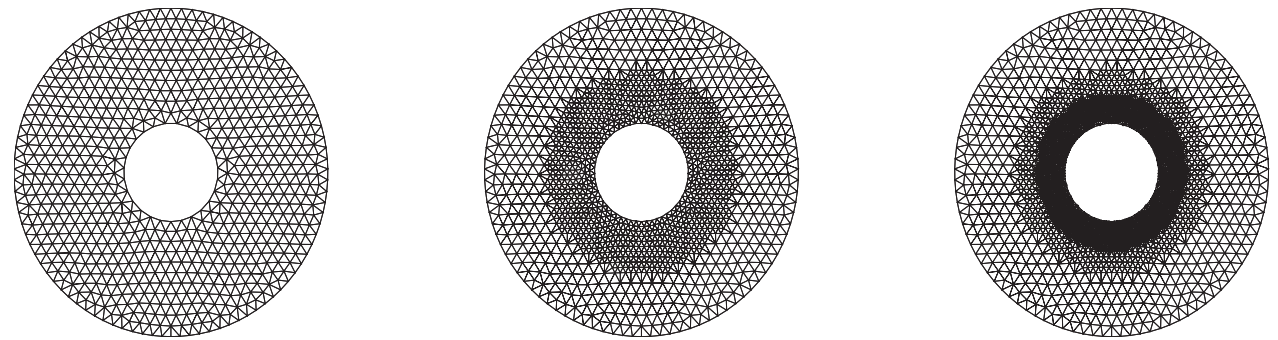

Fig. 10. Mesh refinement for simulation of binary mixture at $260 \mathrm{~K}$ and 70 bar: initial mesh (left), once refined mesh (center), twice refined mesh (right).
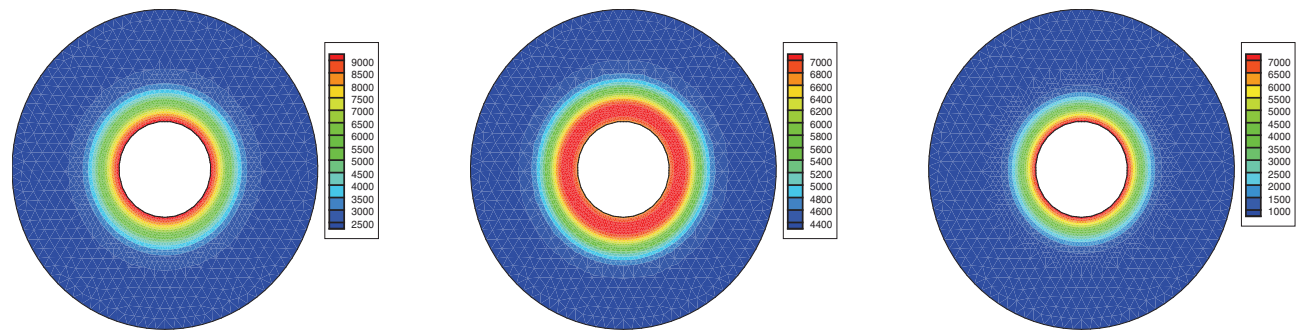

FIG. 11. Binary mixture molar density (in $\mathrm{mol} / \mathrm{m}^{3}$ ) profiles of the two-dimensional problem at $260 \mathrm{~K}$ and 70bar: weighted molar density (left), methane molar density (center), pentane molar density (right).
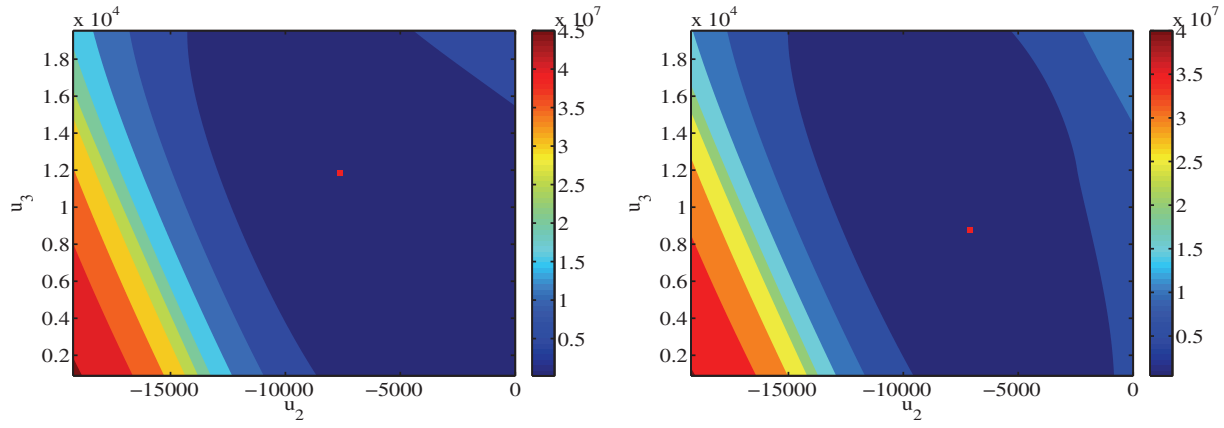

FIG. 12. Grand potential energy profiles of the ternary mixture at 170 bar and different weighted molar densities: $2118 \mathrm{~mol} / \mathrm{m}^{3}$ (left), $2740 \mathrm{~mol} / \mathrm{m}^{3}$ (right); the red dots are the minimum points.

energy as a function of $u_{2}$ and $u_{3}$ for each given $u$. These results also verify the local minimum grand potential energy condition.

Figure 15 depicts the calculated molar density profiles at $300 \mathrm{~K}$ and different pressures. Figure 16 depicts the calculated weighted molar density $(u)$ profiles at $300 \mathrm{~K}$ and different pressures, and the monotonicity of $u$ in the space is also demonstrated. Surface tension computed at 300K and different pressures is illustrated in Figure 17.

For the two-dimensional problem, we simulate this ternary mixture at the temperature $300 \mathrm{~K}$ and the pressure $170 \mathrm{bar}$. The total surface tension calculated on the initial uniform mesh and refined meshes is shown in Figure 18, which also shows the total surface tension decay with the local mesh refinement. Figure 19 illustrates the initial uniform mesh and two refined meshes. Figure 20 depicts the molar density of 

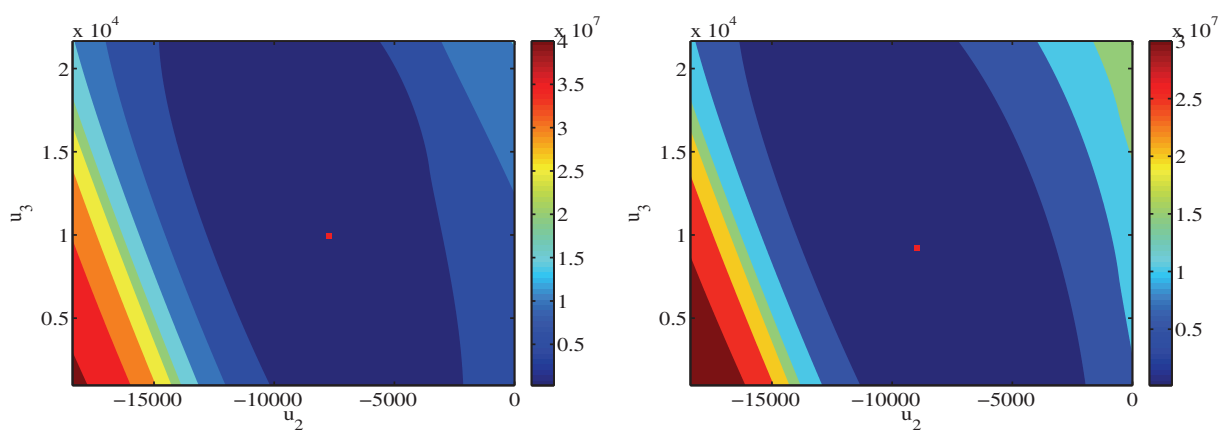

FIG. 13. Grand potential energy profiles of the ternary mixture at 190 bar and different weighted molar densities: $2818 \mathrm{~mol} / \mathrm{m}^{3}$ (left), $3351 \mathrm{~mol} / \mathrm{m}^{3}$ (right); the red dots are the minimum points.
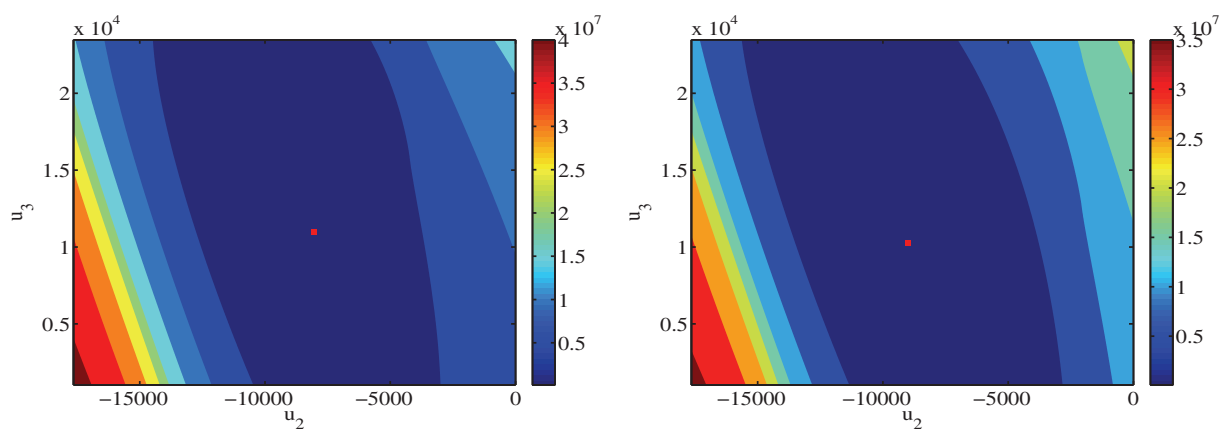

FIG. 14. Grand potential energy profiles of the ternary mixture at 210bar and different weighted molar densities: $2891 \mathrm{~mol} / \mathrm{m}^{3}$ (left), $3330 \mathrm{~mol} / \mathrm{m}^{3}$ (right); the red dots are the minimum points.
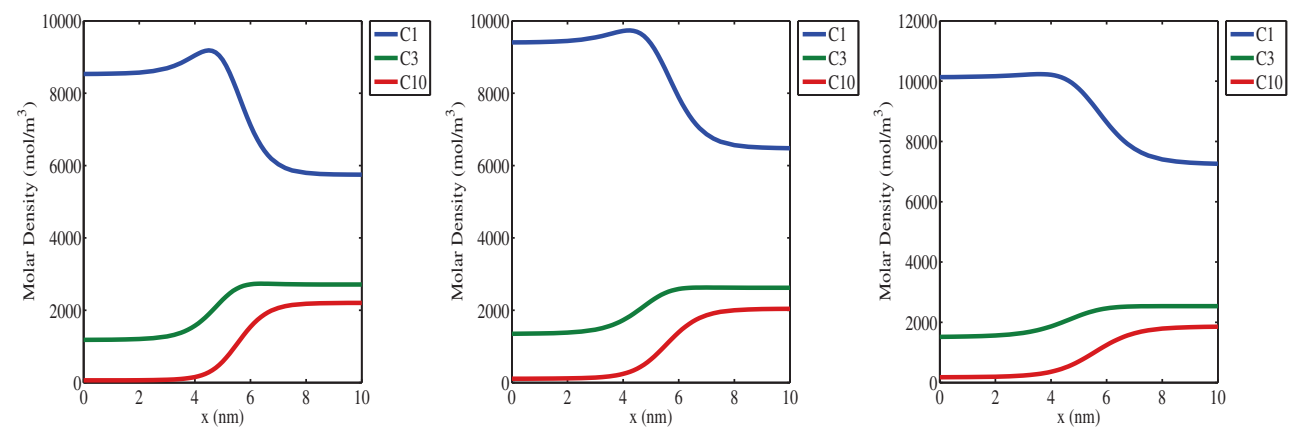

FIG. 15. Ternary mixture molar density profiles at $300 \mathrm{~K}$ and different pressures: $170 \mathrm{bar}$ (left), 190 bar (center), 210bar (right).

each component and the weighted molar density computed on the twice refined mesh. Similar to the binary mixture cases, these results show that the local mesh refinement can also match the mixture composition distribution. The numerical results in both examples indicate that once the surface tension is accurately approximated by using the adaptive mesh-refinement strategy, the mixture composition can also be well simulated, and thus this adaptive method is effective for the numerical simulations of surface tension. 

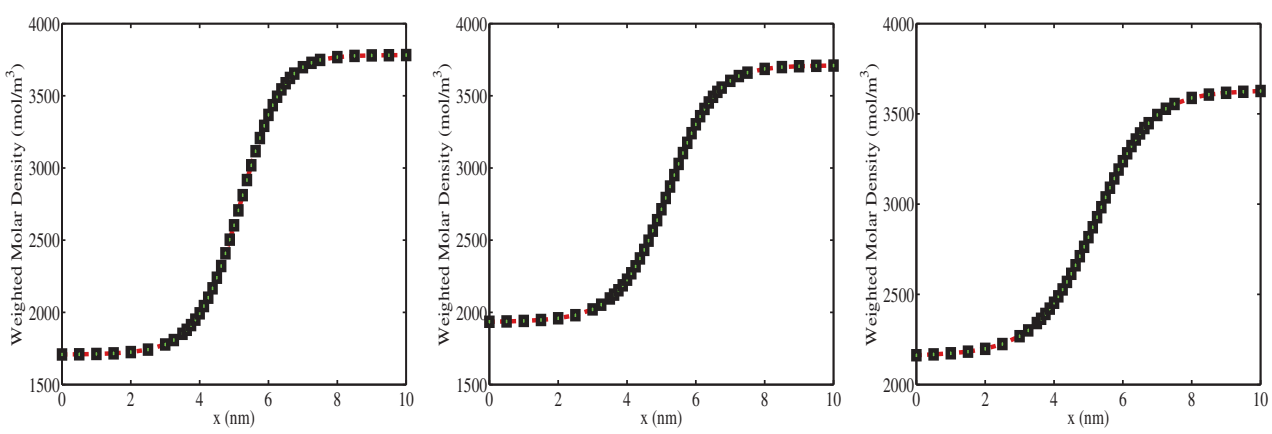

FIG. 16. Ternary mixture weighted molar density profiles at $300 \mathrm{~K}$ and different pressures: 170 bar (left), 190bar (center), 210bar (right).

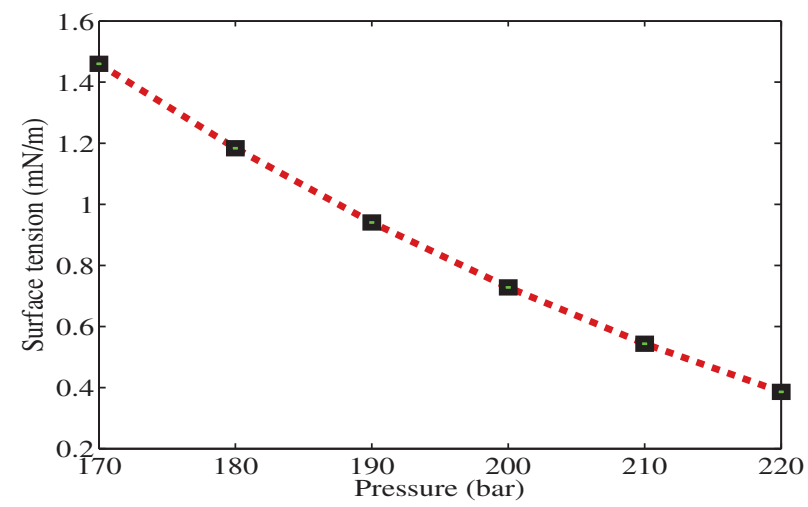

FIG. 17. Surface tension of the ternary mixture at $300 \mathrm{~K}$ and different pressures.

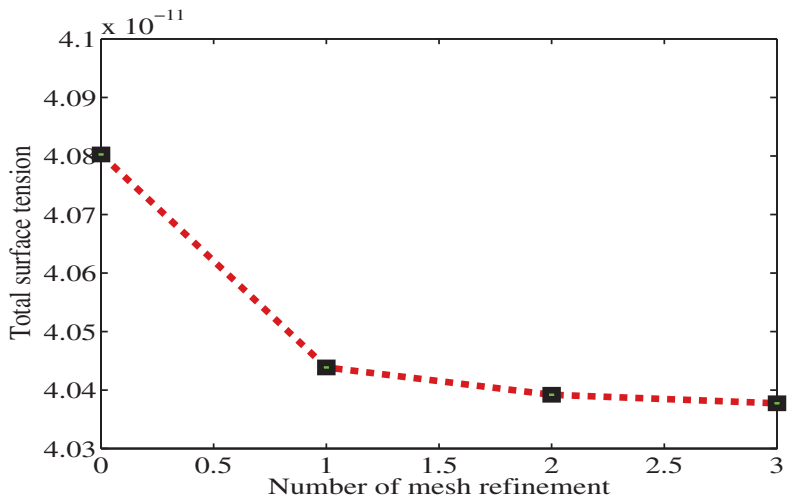

FIG. 18. Total surface tensions of the ternary mixture in the two-dimensional problem at $300 \mathrm{~K}$ and 170 bar.

5. Conclusions. We have studied the multicomponent two-phase interface model with geometric mean influence parameters. By using a linear transformation and the weighted molar density, the formulation of the energy function and the corresponding Euler-Lagrange equations have been reformulated to a conveniently analyzed form. The local minimum grand potential energy condition has been proposed to calculate the mixture compositions for given weighted molar density. The weighted molar 

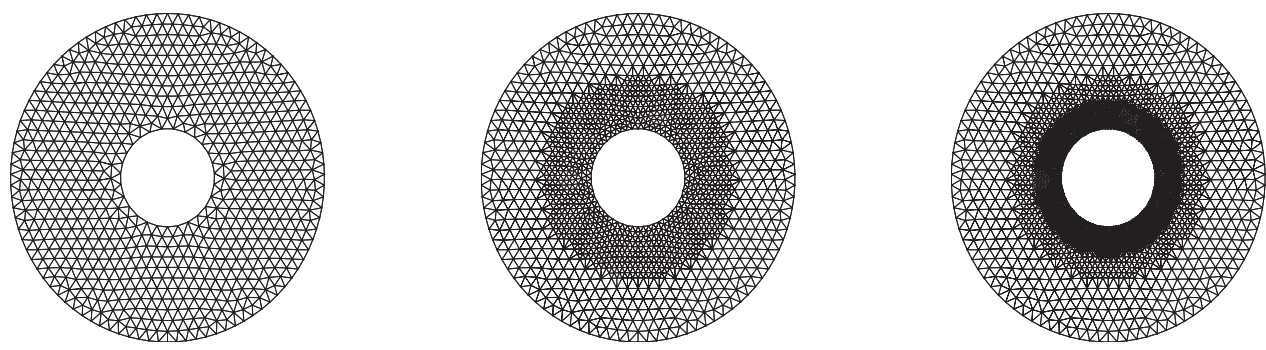

FIG. 19. Mesh refinement for simulation of the ternary mixture at $300 \mathrm{~K}$ and 170bar: initial mesh (left), once refined mesh (center), twice refined mesh (right).

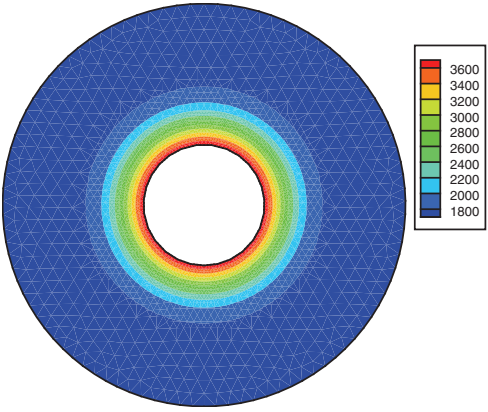

(a)

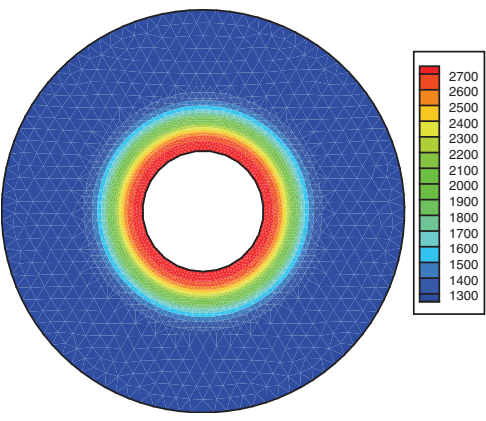

(c)

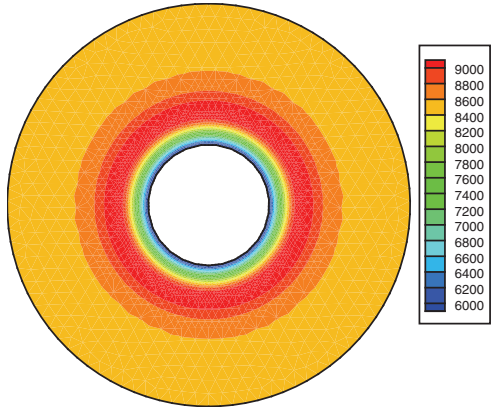

(b)

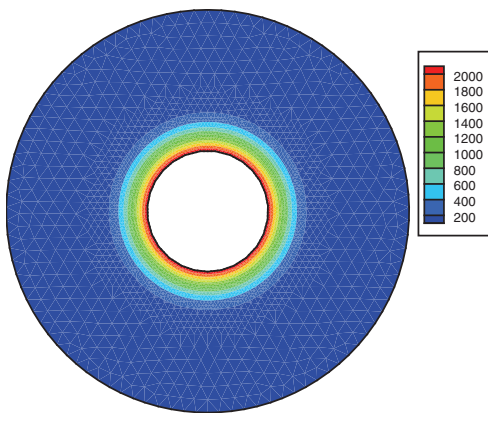

(d)

FIG. 20. Ternary mixture molar density (in $\mathrm{mol} / \mathrm{m}^{3}$ ) profiles of the two-dimensional problem at $300 \mathrm{~K}$ and 170bar: (a) weighted molar density; (b) methane molar density; (c) propane molar density; (d) decane molar density.

density has been proved to satisfy the maximum principle. Thus, we have overcome the key difficulties of theoretical analysis and numerical simulation resulted from the singular influence parameter matrix and the complicated structure of the energy function, and the existence of the solution has been proved under the proper conditions. Based on the property of the problems considered in this paper, we have proposed a modified Newton's method for solving this nonlinear model, and moreover, we have developed and analyzed a physical-based adaptive finite element method. Numerical examples are tested to verify the theoretical results and efficiency of the proposed methods. 
Appendix. The parameters $a(T)$ and $b$ in (1.4) are computed as below. Let $P$ be the pressure of the mixture and let $T_{c_{i}}$ and $P_{c_{i}}$ be critical temperature and pressure, respectively, of the $i$ th component. For the $i$ th component, define the reduced temperature as $T_{r_{i}}=T / T_{c_{i}}$, and furthermore, define the mole fraction $x_{i}=n_{i} / n$, where $n=\sum_{i=1}^{N} n_{i}$. Then $a(T)$ and $b$ are calculated by

$$
a(T)=\sum_{i=1}^{N} \sum_{j=1}^{N} x_{i} x_{j}\left(a_{i} a_{j}\right)^{1 / 2}\left(1-k_{i j}\right), \quad b=\sum_{i=1}^{N} x_{i} b_{i},
$$

where $k_{i j}$ is the given binary interaction coefficients for the energy parameters and

$$
a_{i}=0.45724 \frac{R^{2} T_{c_{i}}^{2}}{P_{c_{i}}}\left[1+m_{i}\left(1-\sqrt{T_{r_{i}}}\right)\right]^{2}, \quad b_{i}=0.07780 \frac{R T_{c_{i}}}{P_{c_{i}}} .
$$

The coefficient $m_{i}$ is calculated by the following formulations:

$$
\begin{aligned}
& m_{i}=0.37464+1.54226 \omega_{i}-0.26992 \omega_{i}^{2}, \quad \omega_{i} \leq 0.49, \\
& m_{i}=0.379642+1.485030 \omega_{i}-0.164423 \omega_{i}^{2}+0.016666 \omega_{i}^{3}, \quad \omega_{i}>0.49,
\end{aligned}
$$

where $\omega_{i}$ is the acentric factor.

The $i$ th component influence parameter $c_{i}$ is calculated as

$$
c_{i}=a_{i} b_{i}^{2 / 3}\left[\alpha_{i}\left(1-T_{r_{i}}\right)+\beta_{i}\right],
$$

where $\alpha_{i}$ and $\beta_{i}$ are the coefficients correlated merely with the acentric factor $\omega_{i}$ of the $i$ th component by the following relations:

$$
\alpha_{i}=-\frac{10^{-16}}{1.2326+1.3757 \omega_{i}}, \quad \beta_{i}=\frac{10^{-16}}{0.9051+1.5410 \omega_{i}} .
$$

The Peng-Robinson equation of state (PR-EOS) [28] is an efficient instrument for modeling the homogeneous fluid and deriving the expression for the Helmholtz energy density required in the gradient theory. The pressure-volume-temperature function of PR-EOS is defined as the following form:

$$
P=\frac{R T}{v-b}-\frac{a(T)}{v(v+b)+b(v-b)}
$$

where $v$ is the molar volume of a mixture.

Acknowledgment. The authors would like to thank the anonymous reviewers for their constructive suggestions and comments to improve the original version of this paper.

\section{REFERENCES}

[1] D.M. Anderson, G.B. McFadden, And A.A. Wheeler, Diffuse-interface methods in fluid mechanics, Ann. Rev. Fluid Mechanics, 30 (1998), pp. 139-165.

[2] I. BABUVŠKA AND W.C. RheinBoldt, Error estimates for adaptive finite element computations, SIAM J. Numer. Anal., 15 (1978), pp. 736-754.

[3] K. BAO, Y. ShI, S. Sun, AND X.-P. WANG, A finite element method for the numerical solution of the coupled Cahn-Hilliard and Navier-Stokes system for moving contact line problems, J. Comput. Phys., 231 (2012), pp. 8083-8099. 
[4] P. BoyanovaA And M. Neytcheva, Efficient numerical solution of discrete multi-component Cahn-Hilliard systems, Comput. Math. Appl., 67 (2014), pp. 106-121.

[5] B. Breure and C.J. Peters, Modeling of the surface tension of pure components and mixtures using the density gradient theory combined with a theoretically derived influence parameter correlation, Fluid Phase Equilibria, 334 (2012), pp. 189-196.

[6] B.S. Carey, L.E. Scriven, and H.T. Davis, Semiempirical theory of surface tension of binary systems, AIChE J., 26 (1980), pp. 705-711.

[7] J.M. Cascon, C. Kreuzer, R.H. Nochetto, and K.G. Siebert, Quasi-optimal convergence rate for an adaptive finite element method, SIAM J. Numer. Anal., 46 (2008), pp. 25242550.

[8] F. Chen And J. Shen, Efficient energy stable schemes with spectral discretization in space for anisotropic Cahn-Hilliard systems, Commun. Comput. Phys., 13 (2013), pp. 1189-1208.

[9] P.M.W. Cornelisse, The Square Gradient Theory Applied-Simultaneous Modelling of Interfacial Tension and Phase Behaviour, Ph.D. thesis, TU Delft, Delft, The Netherlands, 1997.

[10] H.T. Davis, Statistical Mechanics of Phases, Interfaces, and Thin Films, VCH, New York, 1996.

[11] C. Dawson, S. Sun, And M.F. Wheeler, Compatible algorithms for coupled flow and transport, Comput. Methods Appl. Mech. Engrg., 193 (2004), pp. 2565-2580.

[12] V.J. Ervin, E.W. Jenkins, AND S. Sun, Coupled generalized non-linear Stokes flow with flow through a porous medium, SIAM J. Numer. Anal., 47 (2009), pp. 929-952.

[13] A. Firoozabadi, Thermodynamics of Hydrocarbon Reservoirs, McGraw-Hill, New York, 1999.

[14] V. Girault, S. Sun, M.F. Wheeler, And I. Yotov, Coupling discontinuous Galerkin and mixed finite element discretizations using mortar finite elements, SIAM J. Numer. Anal., 46 (2008), pp. 949-979.

[15] H. Gomez And X. Nogueira, An unconditionally energy-stable method for the phase field crystal equation, Comput. Methods Appl. Mech. Engrg., 249 (2012), pp. 52-61.

[16] J. KIM, A continuous surface tension force formulation for diffuse-interface models, J. Comput. Phys., 204 (2005), pp. 784-804.

[17] J. KIM, A generalized continuous surface tension force formulation for phase-field models for multi-component immiscible fluid flows, Comput. Methods Appl. Mech. Engrg., 198 (2009), pp. 3105-3112.

[18] J. KIM, Phase-field models for multi-component fluid flows, Commun. Comput. Phys., 12 (2012), pp. 613-661.

[19] J. Kou AND S. Sun, An adaptive finite element method for simulating surface tension with the gradient theory of fluid interfaces, J. Comput. Appl. Math., 255 (2014), pp. 593-604.

[20] J. Kou, S. Sun, AND X. WANG, Efficient numerical methods for simulating surface tension of multi-component mixtures with the gradient theory of fluid interfaces, Comput. Methods Appl. Mech. Engrg., 292 (2015), pp. 92-106.

[21] J. Kou And S. Sun, Convergence of discontinuous Galerkin methods for incompressible twophase flow in heterogeneous media, SIAM J. Numer. Anal., 51 (2013), pp. 3280-3306.

$[22]$ L.W. Lake, Fundamentals of Enhanced Oil Recovery, Society of Petroleum Engineers, 1986.

[23] K. Mekchay and R.H. Nochetto, Convergence of adaptive finite element methods for general second order linear elliptic PDEs, SIAM J. Numer. Anal., 43 (2005), pp. 1803-1827.

[24] C. Miqueu, B. Mendiboure, C. Graciaa, J. Lachaise, Modelling of the surface tension of binary and ternary mixtures with the gradient theory of fluid interfaces, Fluid Phase Equilibria, 218 (2004), pp. 189-203.

[25] C. Miqueu, B. Mendiboure, A. GraciaA, and J. Lachaise, Modeling of the surface tension of multicomponent mixtures with the gradient theory of fluid interfaces, Industrial Eng. Chem. Res., 44 (2005), pp. 3321-3329.

[26] J. Moortgat, S. Sun, And A. Firoozabadi, Compositional modeling of three-phase flow with gravity using higher-order finite element methods, Water Res. Res., 47 (2011), W05511.

[27] B. Nestler and A. Choudhury, Phase-field modeling of multi-component systems, Curr. Opinion Solid State Mat. Sci., 15 (2011), pp. 93-105.

[28] D. Peng and D.B. Robinson, A new two-constant equation of state, Industrial Eng. Chem. Fund., 15 (1976), pp. 59-64.

[29] Z. QIAO AND S. Sun, Two-phase fluid simulation using a diffuse interface model with PengRobinson equation of state, SIAM J. Sci. Comput., 36 (2014), pp. B708-B728.

[30] M. SAhimi, H. DAVIS, AND L. ScRiven, Thermodynamic modeling of phase and tension behavior of $\mathrm{CO}_{2}$ /hydrocarbon systems, Old SPE Journal, 25 (1985), pp. 235-254.

[31] J. Shen, C. Wang, X. Wang, And S.M. Wise, Second-order convex splitting schemes for gradient flows with Ehrlich-Schwoebel type energy: Application to thin film epitaxy, SIAM J. Numer. Anal., 50 (2012), pp. 105-125.

Copyright (c) by SIAM. Unauthorized reproduction of this article is prohibited. 
[32] J. Shen AND X. YANG, A phase-field model and its numerical approximation for two-phase incompressible flows with different densities and viscosities, SIAM J. Sci. Comput., 32 (2010), pp. 1159-1179.

[33] J. Shen AND X. YAng, Decoupled energy stable schemes for phase-field models of two-phase complex fluids, SIAM J. Sci. Comput., 36 (2014), pp. B122-B145.

[34] P. Sun, C. Liu, AND J. Xu, Phase field model of thermo-induced marangoni effects in the mixtures and its numerical simulations with mixed finite element method, Commun. Comput. Phys., 6 (2009), pp. 1095-1117.

[35] S. Sun And M.F. WheEler, Symmetric and nonsymmetric discontinuous Galerkin methods for reactive transport in porous media, SIAM J. Numer. Anal., 43 (2005), pp. 195-219.

[36] S. Sun And J. Liu, A locally conservative finite element method based on enrichment of the continuous Galerkin method, SIAM J. Sci. Comput., 31 (2009), pp. 2528-2548.

[37] K.E. Teigen, P. Song, J. Lowengrub, And A. Voigt, A diffuse-interface method for twophase flows with soluble surfactants, J. Comput. Phys., 230 (2011), pp. 375-393.

[38] A. VeEser, Convergent adaptive finite elements for the nonlinear Laplacian, Numer. Math., 92 (2002), pp. 743-770.

[39] R. VerfürTh, A review of a posteriori error estimation techniques for elasticity problems, Comput. Methods Appl. Mech. Engrg., 176 (1999), pp. 419-440.

[40] M.F. WheEler, T. Wick, AND W. Wollner, An augmented-Lagrangian method for the phasefield approach for pressurized fractures, Comput. Methods Appl. Mech. Engrg., 271 (2014), pp. 69-85.

[41] H. WU AND Z. CHEN, Uniform convergence of multigrid v-cycle on adaptively refined finite element meshes for second order elliptic problems, Sci. China Ser. A Math., 49 (2006), pp. 1405-1429.

Copyright $@$ ( ) by SIAM. Unauthorized reproduction of this article is prohibited. 